\title{
An implicit potential method along with a meshless technique for incompressible fluid flows for regular and irregular geometries in 2D and 3D
}

\author{
G.C. Bourantas ${ }^{1}$, V.C. Loukopoulos ${ }^{2}$, H.A. Chowdhury ${ }^{3}$, G.R. Joldes ${ }^{3}$, K. Miller ${ }^{3,4}$ and S. \\ P.A. Bordas ${ }^{1,3,4}$ \\ ${ }^{I}$ Faculty of Science, Technology and Communication, University of Luxembourg, \\ Campus Kirchberg, 6, rue Richard Coudenhove-Kalergi L-1359, Luxembourg \\ ${ }^{2}$ Department of Physics, University of Patras, Patras, 26500, Rion, Greece \\ ${ }^{3}$ Intelligent Systems for Medicine Laboratory, \\ School of Mechanical and Chemical Engineering, The University of Western Australia, \\ 35StirlingHighway, Crawley/Perth, WA 6009, Australia \\ ${ }^{4}$ School of Engineering, Cardiff University, The Parade, CF24 3AA Cardiff, United Kingdom
}

\begin{abstract}
We present the Implicit Potential (IPOT) numerical scheme developed in the framework of meshless point collocation. The proposed scheme is used for the numerical solution of the steady state, incompressible Navier-Stokes $(\mathrm{N}-\mathrm{S})$ equations in their primitive variable $(u-v-w-p)$ formulation. The governing equations are solved in their strong form using either a collocated or a semi-staggered type meshless nodal configuration. The unknown field functionsand derivatives are calculated using the Modified Moving Least Squares (MMLS) interpolation method. Both velocitycorrection and pressure-correction methods applied ensure the incompressibility constrain and mass conservation. The proposed meshless point collocation (MPC) scheme has the following characteristics: $(i)$ it can be applied, in a straightforward manner to: steady, unsteady, internal and external fluid flows in 2D and 3D, (ii) it equally applies to regular an irregular geometries, (iii) a distribution of points is sufficient, no numerical integration in space nor any mesh structure are required, (iv) there is no need for pressure boundary conditions since no pressure constitutive equation is solved, $(v)$ it is quite simple and accurate, $(v i)$ results can be obtained using collocated or semi-staggered nodal distributions, (vii) there is no need to compute the velocity potential nor the unit normal vectors and (viii) there is no need for a curvilinear system of coordinates. Simulations of fluid flow in 2D and 3D for regular and irregular geometries indicate the validity of the proposed methodology.
\end{abstract}

Keywords: Implicit potential, Meshless method, Strong form, Incompressible flow, 2D, 3D, MMLS, complementary velocity-pressure.

\section{Introduction}

One of the problems arising in incompressible flow is the explicit treatment of pressure in equations of motion. Moreover, solving numerically theNavier-Stokes $(\mathrm{N}-\mathrm{S})$ equations is a challenging task for a number of reasons. First, and most important, is the inherent non 
linear nature of the partial differential equations. For high velocity or low viscosity the governing equations can produce highly unstable flows (formation of eddies). Second, is the imposition of the incompressibility constraint, with the central question to be answered being the calculation of pressure boundary conditions [1], considering that the governing equations do not provide any boundary conditions for the pressure. Any algorithm developed must ensure a divergence-free flow field at any given time during the calculation.

A significant number of techniques have been developed aiming to deal with the incompressibility constraint [2]. All were successfully incorporated into the traditional mesh-based methods, such as Finite Difference Method (FDM), Finite Element Method (FEM) and Finite Volume Method (FVM). One of the first methods developed using the FDM was the MAC (Marker-and-Cell) scheme, introduced by Harlow and Welch [3]. The MAC scheme is a direct discretization of the (N-S) equations in their primitive variables formulation using second order finite differences on a staggered grid. The convection and viscous terms are solved using explicit time integration, while the pressure term using implicit time integration. Additionally, there is a decoupling ofcomputing the velocity and pressure fields, with the incompressibility constraint being solved on the discretized momentum equation, which results in a discrete Poisson equation forthe pressure. In the late 60s Chorin [4] introduced the projection method that allows simplified treatment of the viscous term. In the context of projection methods an intermediate velocity is computed first and then projected onto the space of incompressible vector fields by solving a Poissontype equation for pressure. The first successful application of FEM to flow problems might be the work of de Vries and Norrie [5], where the Galerkin FEM was applied to incompressible flow with low and moderate Reynolds number. Despite its success, in the cases of high Reynolds numbers the nonlinear convective terms induce numerical oscillations. Consequently, the standard Galerkin FEM, known to be unstable in convection dominated regimes, was modified and new sophisticated methods emerged, such as the streamline upwind Petrov-Galerkin (SUPG) method, the sub-grid scale method, the finite increment calculus (FIC) method, the Taylor-Galerkin (TG) method and the characteristicbased split (CBS) method [6, 7]. In the context of Finite Volume methods frequently used methodologies belong to the Semi-Implicit Method for Pressure Linked Equations 
(SIMPLE) family $[8,9]$. Alternative approaches are the artificial compressibility technique $[10]$ and the Continuity Pressure Vorticity (CVP) method [11, 12]. Therein, the velocity field is corrected according to a well-known vector identity and, on the basis of this correction, the pressure field is subsequently updated. The solution is obtained using the Helmholtz decomposition of the velocity vector and a modified Bernoulli's law for the coupling of the velocity-pressure for the simulation of external flows. In [13] a novel auxiliary potential velocity scheme for incompressible flows was presented, while in [14] the implicit potential method was applied utilizing an implicit potential velocity method for the mass conservation and employing a modified form of Bernoulli's law for the coupling of the velocity-pressure corrections. When a potential velocity is introduced, where the velocity correction is applied in order to fulfil continuity equation, an additional equation for the potential of the velocity is introduced. The BCs for the velocity-correction potential function require the computation of the unit normal vectors. It is usually a difficult task, especially in the case of irregular geometries. In the proposed scheme there is no need to compute a potential velocity and unit normal vectors.

Both FDM and FVM methods widely use a semi-staggered or a fully staggered grid, applied in flow problems with uniform spatial domain or with some kind of symmetry. Although the applicability of the method in irregular geometries is feasible, the computational cost may increase drastically. On the other hand, mesh-based methods, despite their success, have some serious drawbacks related to the mesh generation. Mesh generation is still a difficult task, especially for3D geometries, being the bottleneck of the entire simulation procedure. The main drawback is the refinement process. Eventually, meshless methods have recently emerged as a possible alternative to overcome the problems of mesh generation and facilitate local refinement of the approximation scheme.

In the context of Meshless methods (MM) the spatial domain is represented by using a set of nodes, uniformly or randomly distributed along the interior and on the boundaries, without any inter-connectivity. A practical overview of meshless methods based on global weak forms was given in [15]. Numerous MMs schemes were developed in both Eulerian and Lagrangian frameworks, such as the Meshless Local Petrov-Galerkin (MLPG) [1621], Local Boundary Integral Equation (LBIE) [22, 23], Meshless Point Collocation (MPC) [24-28], Element Free Galerkin (EFG) [29, 30], Smoothed Particle Hydrodynamics (SPH) 
[31-33] and Finite Point method [34-36], applied on the numerical solution of (N-S) equations. Flow equations can be solved in their primitive variables formulation or in their velocity-vorticity and stream function-vorticity formulation. In the majority of these methods the calculation of pressure was done explicitly or as a final outcome, given the boundary conditions for pressure.

The present study deals with the reformulation of the implicit potential (IPOT) methodology [14] and its application in the context of meshless methods. The proposed scheme solves numerically the steady state, laminar and incompressible (N-S) equations, in their primitive variables formulation using a collocated or semi-staggered nodal arrangement. The novelty relies on the introduction of a complementary pressure (pressure correction) through the introduction of a complementary velocity, which ensures mass conservation. Moreover, we suppose that the complementary velocity and pressure correspond to a complementary flow. Consequently, an "appropriate" momentum equation appears, which can be described as a modified expression of Bernoulli's law for the complementary flow and, after some algebra, the complementary pressure is obtained. In fact, both pressures, complementary and physical, are calculated through an algebraic relation without solving any partial differential equation. Eventually, the number of equations solved decreases. To the authors' knowledge, this is the first attempt to apply the proposed IPOT methodology using meshless schemes in general and, specifically, the MMLS method, to approximate the flow variables. The proposed IPOT meshless point collocation (MPC) scheme has the following characteristics: (i) it can be applied, in a straightforward manner, to steady, unsteady, internal and external fluid flows in 2D and 3D, (ii) it is equally performant for regular an irregular geometries, (iii) a distribution of points is sufficient, no numerical integration in space nor any mesh structure is required, (iv) there is no need for pressure boundary conditions since no pressure constitutive equation is solved, (v) it is quite simple and accurate, (vi) results can be obtained using collocated or semi-staggered nodal distributions, (vii) there is no need neither for the computation of the velocity potential nor the computation of the unit normal vectors and (viii) there is no need for a curvilinear system of coordinates.

The rest of the paper is organized as follows. In Section 2, the governing equations along with the proposed IPOT numerical method are presented, while the approximation method 
of the classical Moving Least Squares (MLS) and the Modified MLSare briefly presented in Section 3. Section 4 presents the verification benchmark flow problems used along with the test casesused to demonstrate and highlight the accuracy, robustness, and computational efficiency of the proposed scheme. Finally, the conclusions are given in Section 5.

\section{Governing equations and solution procedure}

\subsection{Governing equations}

Navier-Stokes equations express conservation of linear momentum. They are a set of nonlinear partial differential equations (PDEs) which, in velocity-pressure formulation [2],can be written in non-dimensional form as:

- Momentum equation

$$
(\boldsymbol{u} \cdot \nabla) \boldsymbol{u}=-\nabla p+\frac{1}{R e} \nabla^{2} \boldsymbol{u}+\boldsymbol{F}
$$

- Continuity equation

$$
\boldsymbol{\nabla} \cdot \boldsymbol{u}=0
$$

where $\boldsymbol{u}$ is the flow velocity vector, $p$ is the pressure field, $R e$ is the Reynolds number and $\boldsymbol{F}$ corresponds to body force terms (herein we assume $\boldsymbol{F}=\mathbf{0}$ ). All field variables are functions of space, $\boldsymbol{x}$, in a fixed domain $\Omega$ surrounded by a closed boundary. The system of PDEs (1)-(2) is closed with appropriate boundary conditions (BCs) related to the physical problem considered. Different types of BCs can be used, namely Dirichlet, Neumann, Robin, mixed type etc. In the present paper the applied boundary conditions are described in the numerical examples examined.

\subsection{Solution procedure with IPOT scheme}


In the context of the strong form meshless point collocation method, an iterative scheme has been developed for the numerical solution of the (N-S) equations in their primitive variables (velocity-pressure) formulation. The nonlinear PDEs were linearized using the lagging of coefficients method [26].

The iterative procedure initiates by setting pressure $\left(p^{(n)}\right)$ and velocity $\left(\boldsymbol{u}^{(n)}\right)$ field values at the $n^{\text {th }}$ iteration on the pressure and velocity nodes, respectively. Afterwards, momentum equations aresolved to give an estimation of the velocity field $\boldsymbol{u}^{(n+1)}$ (next iteration). The governing equations at the $(n+1)$ iteration become

$\left(\boldsymbol{u}^{(n)} \cdot \nabla\right) \boldsymbol{u}^{(n+1)}=-\nabla p^{(n+1)}+\frac{1}{R e} \nabla^{2} \boldsymbol{u}^{(n+1)}$.

$\boldsymbol{\nabla} \cdot \boldsymbol{u}^{(n+1)}=0$.

The estimated velocity field $\boldsymbol{u}^{(n+1)}$ does not necessarily satisfy the continuity equation $\left(\boldsymbol{\nabla} \cdot \boldsymbol{u}^{(n+1)} \neq 0\right)$. Hence, a complementary velocity $\left(\boldsymbol{u}_{a}^{(n+1)}\right)$ is introduced, given by

$\boldsymbol{u}_{c}^{(n+1)}=\boldsymbol{u}^{(n+1)}+\boldsymbol{u}_{a}^{(n+1)}$,

with $\boldsymbol{u}_{c}^{(n+1)}$ being the corrected velocity value. The corrected velocity satisfies the continuity equation by definition; the following equation can be written for the complementary velocity

$\boldsymbol{\nabla} \cdot \boldsymbol{u}_{c}^{(n+1)}=0 \Leftrightarrow \boldsymbol{\nabla} \cdot\left(\boldsymbol{u}^{(n+1)}+\boldsymbol{u}_{a}^{(n+1)}\right)=0 \Leftrightarrow \boldsymbol{\nabla} \cdot \boldsymbol{u}_{a}^{(n+1)}=-\boldsymbol{\nabla} \cdot \boldsymbol{u}^{(n+1)}$.

The complementary velocity vector can be written as the sum of a curl vector function and a divergence vector function (Helmholtz decomposition):

$\boldsymbol{u}_{a}^{(n+1)}=\left(\boldsymbol{u}_{a}^{(n+1)}\right)_{\text {irrot }}+\left(\boldsymbol{u}_{a}^{(n+1)}\right)_{\text {solen }}$ 
which is the sum of an irrotational term for which $\boldsymbol{\nabla} \times\left(\boldsymbol{u}_{a}^{(n+1)}\right)_{\text {irrot }}=\mathbf{0}$ and a solenoidal term for which $\boldsymbol{\nabla} \cdot\left(\boldsymbol{u}_{a}^{(n+1)}\right)_{\text {solen }}=0$. The solenoidal component of the complementary velocity does not affect the mass conservation equation and can be ignored. Thus, the complementary velocity can, at the same time, satisfy the continuity equation and be an irrotational vector $\boldsymbol{u}_{a}^{(n+1)}=\left(\boldsymbol{u}_{a}^{(n+1)}\right)_{\text {irrot }}$. In fact this does not alter the nature of the flow, in terms of the velocity field, since the complementary velocity is introduced to impose mass conservation rather than to correct the velocity field. Moreover, the complementary velocity field can be given as $\boldsymbol{u}_{a}^{(n+1)}=\boldsymbol{u}^{(n+1)}-\boldsymbol{u}^{(n)}$, since the velocity values at $n^{\text {th }}$ and $(n+1)^{\text {th }}$ iterations slightly differ (are of the same order of magnitude).

For the complementary pressure, first we assume that the complementary momentum equation is satisfied

$$
\left(\boldsymbol{u}^{(n)} \cdot \boldsymbol{\nabla}\right) \boldsymbol{u}_{a}^{(n+1)}=-\nabla p_{a}^{(n+1)}+\frac{1}{R e_{a}} \nabla^{2} \boldsymbol{u}_{a}^{(n+1)}
$$

which conceptually corresponds to creeping flow with $R e_{a} \approx O\left(10^{0}\right)$. Second, we express the convection terms of the complementary momentum equation according to the vector identity

$$
\begin{aligned}
\boldsymbol{\nabla} \cdot\left(\boldsymbol{u}^{(n)} \cdot \boldsymbol{u}_{a}^{(n+1)}\right. & ) \\
& =\left(\boldsymbol{u}^{(n)} \cdot \boldsymbol{\nabla}\right) \boldsymbol{u}_{a}^{(n+1)}+\left(\boldsymbol{u}_{a}^{(n+1)} \cdot \boldsymbol{\nabla}\right) \boldsymbol{u}^{(n)}+\boldsymbol{u}^{(n)} \times\left(\boldsymbol{\nabla} \times \boldsymbol{u}_{a}^{(n+1)}\right) \\
& +\boldsymbol{u}_{a}^{(n+1)} \\
& \times\left(\boldsymbol{\nabla} \times \boldsymbol{u}^{(n)}\right) .
\end{aligned}
$$

The complementary momentum equation (Eq. (8)) at $(n+1)$ iteration becomes

$$
\frac{1}{2} \boldsymbol{\nabla} \cdot\left(\boldsymbol{u}^{(n)} \cdot \boldsymbol{u}_{a}^{(n+1)}\right)-\boldsymbol{u}^{(n)} \times\left(\boldsymbol{\nabla} \times \boldsymbol{u}_{a}^{(n+1)}\right)+\nabla p_{a}^{(n+1)}-\nabla^{2} \boldsymbol{u}_{a}^{(n+1)}=\boldsymbol{R}_{a}^{(n+1)}
$$


where

$$
\begin{gathered}
\boldsymbol{R}_{a}^{(n+1)}=-\frac{1}{2} \boldsymbol{\nabla}\left(\boldsymbol{u}^{(n)} \cdot \boldsymbol{u}_{a}^{(n+1)}\right)+\left(\boldsymbol{u}_{a}^{(n+1)} \cdot \boldsymbol{\nabla}\right) \boldsymbol{u}^{(n)}+\boldsymbol{u}_{a}^{(n+1)} \times\left(\boldsymbol{\nabla} \times \boldsymbol{u}^{(n)}\right) \\
=\frac{1}{2} \sum_{i=1}^{3}\left(u_{i, a}^{(n+1)} \nabla u_{i}^{(n)}-u_{i}^{(n)} \nabla u_{i, a}^{(n+1)}\right) .
\end{gathered}
$$

and $u_{i}(i=1,2,3)$ stands for the three components of the velocity, while $u_{i, \alpha}(i=1,2,3)$ stands for the three components of the complementary velocity. The term $\boldsymbol{R}_{a}^{(n+1)}$ is a byproduct of the linearization of the convection terms. Additionally, when $\boldsymbol{u}_{a}^{(n+1)}-\boldsymbol{u}_{a}^{(n)} \rightarrow 0$ the term $\boldsymbol{R}_{a}^{(n+1)} \rightarrow 0$. Moreover, an order of magnitude analysis showed that this term is negligible compared to other terms [13]. Therefore, we neglect the term $\boldsymbol{R}_{a}^{(n+1)}$, which expresses the error in the estimation of the convection term correction due to the lagging of the nonlinear term. This term reduces to zero as the numerical scheme converges and does not affect the accuracy of Eq. (10) or (8). Finally, we obtain the conservative form of Eq. (8)

$$
\frac{1}{2} \boldsymbol{\nabla} \cdot\left(\boldsymbol{u}^{(n)} \cdot \boldsymbol{u}_{a}^{(n+1)}\right)-\boldsymbol{u}^{(n)} \times\left(\boldsymbol{\nabla} \times \boldsymbol{u}_{a}^{(n+1)}\right)+\nabla p_{a}^{(n+1)}-\nabla^{2} \boldsymbol{u}_{a}^{(n+1)}=0 .
$$

The complementary velocity function $\boldsymbol{u}_{\alpha}$ is always irrotational $\left(\boldsymbol{\nabla} \times \boldsymbol{u}_{a}^{(n+1)}=\mathbf{0}\right)$ and independent of the nature of the flow. Additionally, it can be linked to a complementary pressure function $p_{\alpha}$ through a "complementary" momentum equation. By utilizing the following vector identity

$$
\nabla^{2} \boldsymbol{u}_{a}^{(n+1)}=\boldsymbol{\nabla}\left(\boldsymbol{\nabla} \cdot \boldsymbol{u}_{a}^{(n+1)}\right)-\boldsymbol{\nabla} \times\left(\boldsymbol{\nabla} \times \boldsymbol{u}_{a}^{(n+1)}\right)=\boldsymbol{\nabla}\left(\boldsymbol{\nabla} \cdot \boldsymbol{u}_{a}^{(n+1)}\right),
$$

Eq. (12) can be written as

$$
\boldsymbol{\nabla}\left(\frac{1}{2} \boldsymbol{u}^{(n)} \cdot \boldsymbol{u}_{a}^{(n+1)}+p_{a}^{(n+1)}-\boldsymbol{\nabla} \cdot \boldsymbol{u}_{a}^{(n+1)}\right)=0
$$


and Eq. (14) gives

$\frac{1}{2} \boldsymbol{u}^{(n)} \cdot \boldsymbol{u}_{a}^{(n+1)}+p_{a}^{(n+1)}-\boldsymbol{\nabla} \cdot \boldsymbol{u}_{a}^{(n+1)}=$ constant $=0$

In Eq. (15) the arbitrary constant should be zero when the continuity equation is satisfied and $\boldsymbol{u}_{a}^{(n+1)}=0, p_{a}^{(n+1)}=0$. Using Eq. (6) in Eq. (15), the following formula for the complementary pressure is obtained:

$p_{a}^{(n+1)}=-\frac{1}{2} \boldsymbol{u}^{(n)} \boldsymbol{u}_{a}^{(n+1)}-\boldsymbol{\nabla} \cdot \boldsymbol{u}^{(n+1)}$.

In this way we evaluate complementary pressure function $p_{a}^{(n+1)}$ without ignoring the first term of Eq. (8) as it has been implemented in [14]. Nevertheless, a simplified version of Eq. (16) can be obtained if the convection term of Eq. (8) is neglected and the first term of the right side of Eq. (16) is eliminated. The complementary velocity function $\boldsymbol{u}_{a}^{(n+1)}$ is of the same order of magnitude with $\boldsymbol{u}^{(n+1)}-\boldsymbol{u}^{(n)}$ and we can estimate it without solving an additional PDE. Finally, the update pressure field is given by

$p^{(n+1)}=p^{(n)}+p_{a}^{(n+1)}$

\subsection{Algorithmic description of the implicit potential method}

The algorithmic steps of describing the method can be summarized as follows:

- For an initial pressure and velocity field $p^{(n)}$ and $\boldsymbol{u}^{(n)}$, we solve the momentum equation (Eq. (3)) to obtain the updated velocity field $\boldsymbol{u}^{(n+1)}$.

- Using the computed velocity field $\boldsymbol{u}^{(n+1)}$ and the complementary velocity function $\boldsymbol{u}_{a}^{(n+1)}$, compute the complementary pressure function $p_{a}^{(n+1)}$ given by Eq. (16). 
- Obtain the updated pressure field $p^{(n+1)}$ using Eq.(17) and proceed to the next iteration.

The convergence of the proposed scheme was monitored as $\left|\frac{g^{(n+1)}-g^{(n)}}{g^{(n+1)}}\right| \leq 10^{-5}$, where $g$ stands for every unknown function $(u, v, w, p)$ and $n$ represents the $n^{\text {th }}$ iteration. As soon as the convergence criteria are satisfied, the iterative procedure ends; otherwise, the current velocity and pressure values are replaced with the updated ones and the procedure is repeated. The linear systems, obtained from the discretization of the PDEs of the flow, are solved using a direct Gauss elimination method. Additionally, other iterative linear equations solvers, as the biconjugate gradient method, were applied, demonstrating the accuracy of the numerical procedure.

\section{Meshless approximation scheme}

We present the classical MLS and the Modified MLS method used in this paper.

\subsection{Moving Least Squares (MLS)}

MLS is one ofthe most widely used meshless approximation schemes [37]. Consider a set of $N$ nodes scattered in the domain $\Omega$ and $\boldsymbol{x}_{i}$ the coordinates of the node $i$. The function $u(\boldsymbol{x})$ around a point $\boldsymbol{x}$ is locally approximated by the function $u^{h}(\boldsymbol{x})$, which can be expressed as

$u^{h}(\boldsymbol{x})=\sum_{i=1}^{m} p_{i}(\boldsymbol{x}) a_{i}(\boldsymbol{x})=\mathbf{p}^{T}(\boldsymbol{x}) \mathbf{a}(\boldsymbol{x})$,

where $m$ is the number of terms in the basis, $\boldsymbol{p}(\boldsymbol{x})$ is a polynomial basis in the space coordinates that consists of monomials of the lowest order to ensure completeness (herein we consider a second order polynomial, thus $m=6$ in $2 \mathrm{D}$ and $m=10$ in $3 \mathrm{D})$ and $a_{i}(\boldsymbol{x})$ are constants which, as indicated, are functions of the spatial co-ordinates $\boldsymbol{x}$. Due to the local nature of the approximation, the polynomial basis can be written in $2 \mathrm{D}$ as 
$\boldsymbol{p}^{T}\left(\boldsymbol{x}-\boldsymbol{x}_{i}\right)=\left[1,\left(x-x_{i}\right),\left(y-y_{i}\right),\left(x-x_{i}\right)^{2},\left(x-x_{i}\right)\left(y-y_{i}\right),\left(y-y_{i}\right)^{2}\right]$,

and in $3 \mathrm{D}$ as

$$
\begin{aligned}
\boldsymbol{p}^{T}\left(\boldsymbol{x}-\boldsymbol{x}_{i}\right)= & {\left[1,\left(x-x_{i}\right),\left(y-y_{i}\right),\left(z-z_{i}\right),\left(x-x_{i}\right)^{2},\left(y-y_{i}\right)^{2},\left(z-z_{i}\right)^{2},\left(x-x_{i}\right)(y\right.} \\
& \left.\left.-y_{i}\right),\left(x-x_{i}\right)\left(y-y_{i}\right),\left(y-y_{i}\right)\left(z-z_{i}\right)\right] .
\end{aligned}
$$

The coefficients $a_{i}(\boldsymbol{x})$ are obtained by performing a weighted least square fit for the localapproximation, which is obtained by minimising the difference between the local approximation andthe function. The fit of function $u(x)$ to the data values $u_{1}, u_{2}, \ldots, u_{N}$ can be evaluated by means of the error functional $\boldsymbol{J}(\boldsymbol{x})$ given as

$$
\begin{array}{r}
\boldsymbol{J}(x)=\sum_{j=1}^{N}\left(u^{h}\left(x_{j}\right)-u_{j}\right)^{2} w\left(\left\|\boldsymbol{x}-\boldsymbol{x}_{j}\right\|\right) \\
=\sum_{j=1}^{N}\left(p^{T}\left(x_{j}\right) a\left(x_{j}\right)-u_{j}\right)^{2} w\left(\left\|\boldsymbol{x}-\boldsymbol{x}_{j}\right\|\right)
\end{array}
$$

where $w\left(\left\|\boldsymbol{x}-\boldsymbol{x}_{j}\right\|\right)$ is a weight function with compact support. The weight function depends notonly on data points $\boldsymbol{x}_{j}$; they also are functionsof $\boldsymbol{x}$, the location in the spatial domain $\Omega$ where a fit is required. The weightfunctionshave relatively large values for points $\boldsymbol{x}_{j}$ close to $\boldsymbol{x}$, and relatively small for the more distant points $\boldsymbol{x}_{j}$. The following choice of the weight function $w\left(\left\|\boldsymbol{x}-\boldsymbol{x}_{j}\right\|\right)$ was used here

$w\left(\left\|\boldsymbol{x}-\boldsymbol{x}_{j}\right\|\right)=w\left(d_{j}\right)=\left\{\begin{array}{cc}e^{-\left(\frac{d_{j}}{a_{0} h}\right)^{2}} & \text { inside support domain } \\ 0 & \text { outside support domain }\end{array}\right.$

where $d_{i}=\left\|\boldsymbol{x}-\boldsymbol{x}_{j}\right\|$ and $a_{0}$ is a prescribed constant, defined as a fraction of the radius of the support domain $h$. The value of $a_{0}$ has to be predefined, usually with the help of numerical experiments for the same class of problemswhere solutions already exist. A value between 0.2 and 0.3 gives satisfactory approximation of the field function. The value $a_{0}=0.2$ was used here, which was found to reproduce with excellent accuracy verified numerical solutions of benchmark problems (see Section 4).

Eq. (21) can be rewritten in the form 
$J(x)=(\mathbf{P a}-\mathbf{u})^{T} \mathbf{W}(\boldsymbol{x})(\mathbf{P a}-\mathbf{u})$

where

$$
\begin{aligned}
& u^{T}=\left(u_{1}, u_{2}, \ldots, u_{N}\right) \\
& \mathbf{P}=\left[\begin{array}{c}
\boldsymbol{p}^{T}\left(\boldsymbol{x}_{1}\right) \\
\ldots \\
\boldsymbol{p}^{T}\left(\boldsymbol{x}_{n}\right)
\end{array}\right] \\
& \mathbf{P}^{T}\left(\boldsymbol{x}_{j}\right)=\left(p_{1}\left(\boldsymbol{x}_{j}\right), \ldots, p_{m}\left(\boldsymbol{x}_{j}\right)\right)(26) \\
& \boldsymbol{W}=\operatorname{diag}\left(w\left(\boldsymbol{x}-\boldsymbol{x}_{1}\right), \ldots, w\left(\boldsymbol{x}-\boldsymbol{x}_{n}\right)\right)
\end{aligned}
$$

To compute the coefficients $\boldsymbol{a}(\boldsymbol{x})$, we minimize function $\boldsymbol{J}$ with respect to coefficients $\boldsymbol{a}(\boldsymbol{x})$

$\frac{\partial \boldsymbol{J}}{\partial \boldsymbol{a}}=\mathbf{A}(\boldsymbol{x}) \mathbf{a}(\boldsymbol{x})-\mathbf{B}(\boldsymbol{x}) \mathbf{u}=\mathbf{0}$

where

$$
\begin{aligned}
\mathbf{A}(\boldsymbol{x}) & =\mathbf{P}^{T} \mathbf{W}(\boldsymbol{x}) \mathbf{P}=\sum_{i=1}^{N} w_{i}\left(\boldsymbol{x}-\boldsymbol{x}_{i}\right) \boldsymbol{p}\left(\boldsymbol{x}_{i}\right) \boldsymbol{p}^{T}\left(\boldsymbol{x}_{i}\right), \\
\mathbf{B}(\boldsymbol{x}) & =\mathbf{P}^{T} \mathbf{W}(\boldsymbol{x})=\left[w_{1}(\boldsymbol{x}) \boldsymbol{p}\left(\boldsymbol{x}_{1}\right), w_{2}(\boldsymbol{x}) \boldsymbol{p}\left(\boldsymbol{x}_{2}\right), \ldots, w_{N}(\boldsymbol{x}) \boldsymbol{p}\left(\boldsymbol{x}_{N}\right)\right]
\end{aligned}
$$

and therefore

$$
\mathbf{a}(\boldsymbol{x})=\mathbf{A}^{-1}(\boldsymbol{x}) \mathbf{B}(\boldsymbol{x}) \mathbf{u} .
$$

Finally, the dependent variable $u^{h}(\boldsymbol{x})$ can then be expressed as

$$
u^{h}(\boldsymbol{x})=\sum_{I=1}^{N} \Phi_{I}(\boldsymbol{x}) u_{I}
$$

with $u_{I}$ is the value of the field $u$ at $\boldsymbol{x}_{I}$ and $\Phi_{I}$ is the shape function of the node $I$, given by 
$\Phi_{i}(\boldsymbol{x})=\boldsymbol{p}^{T}(\boldsymbol{x}) \boldsymbol{A}^{-1}(\boldsymbol{x}) \boldsymbol{p}\left(\boldsymbol{x}_{i}\right) w_{i}(\boldsymbol{x})$.

The partial derivatives of the shape functions $\Phi_{I}(\boldsymbol{x})$ are obtained as

$\Phi_{I, k}(x)=\sum_{j=1}^{m}\left[p_{j, k}\left(\boldsymbol{A}^{-1} \boldsymbol{B}\right)_{j i}+p_{j}\left(\boldsymbol{A}^{-1} \boldsymbol{B}_{, \boldsymbol{k}}+\boldsymbol{A}_{, \boldsymbol{k}}^{-\mathbf{1}} \boldsymbol{B}\right)_{j i}\right]$,

with ( ),$k$ denotes differentiation with respect to $k$ and, $\boldsymbol{A}_{, \boldsymbol{k}}^{\boldsymbol{- 1}}$ represents the derivative of the inverse of matrix A given by

$A_{, \mathrm{k}}^{-1}=-A^{-1} A_{, \mathrm{k}} A^{-1}$

\subsection{Modified Moving Least Squares (MMLS)}

The advantage of Modified MLS comes to the computation of the moment matrix A. A singular moment matrix mathematically means that the functional

$\boldsymbol{J}(x)=\sum_{j=1}^{n}\left[\left(u^{h}\left(x_{j}\right)-u_{j}\right)^{2} w\left(\left\|\boldsymbol{x}-\boldsymbol{x}_{j}\right\|\right)\right]$

used to compute the coefficients $\boldsymbol{a}(\boldsymbol{x})$ in MLS, has multiple solutions, and therefore it does not include sufficient constraints to guarantee a unique solution for the given node distribution. Based on this fact, authors in [38] proposed to add additional constraints to the functional $\boldsymbol{J}^{M M L S}$ as follows:

$\boldsymbol{J}^{\boldsymbol{M} \boldsymbol{M L S}}(x)=\sum_{j=1}^{n}\left[\left(u^{h}\left(x_{j}\right)-u_{j}\right)^{2} w\left(\left\|\boldsymbol{x}-\boldsymbol{x}_{j}\right\|\right)\right]+\mu_{x^{2}} a_{x^{2}}^{2}+\mu_{x y} a_{x y}^{2}+\mu_{y^{2}} a_{y^{2}}^{2}$

with 
$\boldsymbol{\mu}=\left[\begin{array}{lll}\mu_{x^{2}} & \mu_{x y} & \mu_{y^{2}}\end{array}\right]$

is a vector of positive weights for the additional constraints (for simplicity, the derivation is only presented for the $2 \mathrm{D}$ case).

The choice of the additional constraints ensures that, when the classical MLS moment matrix is singular (multiple solutions), we obtain the solution having the coefficients for the higher order monomials in the bases equal to zero. By choosing the additional weights as small positive numbers, we can ensure that the classical MLS solution is altered only very slightly when the moment matrix is not singular. This has already been demonstrated in the case of solids undergoing finite deformation [39].

The new functional can be rewritten in matrix form as

$\boldsymbol{J}^{M M L S}=(\boldsymbol{P a}-\boldsymbol{u})^{T} \boldsymbol{W}(\boldsymbol{P a}-\boldsymbol{u})+\boldsymbol{a}^{T} \boldsymbol{H a}$,

where $\boldsymbol{H}$ is a matrix with all elements equal to zero except the last 3 diagonal entries, which are equal to $\mu$ :

$\boldsymbol{H}=\left[\begin{array}{cc}\mathbf{0}_{33} & \mathbf{0}_{33} \\ \mathbf{0}_{33} & \operatorname{diag}(\boldsymbol{\mu})\end{array}\right]$.

Then, following the minimization procedure for the functional $\boldsymbol{J}^{M M L S}$ used in the classical MLS we get

$\frac{\partial J^{M M L S}}{\partial a}=\left[\boldsymbol{P}^{T} \boldsymbol{W}+\boldsymbol{H}\right] \boldsymbol{a}(\boldsymbol{x})-\boldsymbol{P}^{T} \boldsymbol{W u}=0$.

For the modified moment matrix we can write

$M^{M M L S}=\boldsymbol{P}^{T} W P+H=M+H$,

while the new coefficients can be computed as 
$a(x)=\left(P^{T} W P+H\right)^{-1} P^{T} W u$.

Finally, the modified approximant becomes

$u^{M M L S}(\boldsymbol{x})=\boldsymbol{P}^{\boldsymbol{T}}\left(\boldsymbol{P}^{\boldsymbol{T}} \boldsymbol{W} \boldsymbol{P}+\boldsymbol{H}\right)^{-1} \boldsymbol{P}^{T} \boldsymbol{W} \boldsymbol{u}=\sum_{j=1}^{n} \Phi_{j}^{M M L S}(\boldsymbol{x}) u_{j}$,

with the new shape functions

$\boldsymbol{\Phi}^{M M L S}(\boldsymbol{x})=\left[\Phi_{1}^{M M L S}(\boldsymbol{x}) \quad \ldots \quad \Phi_{n}^{M M L S}(\boldsymbol{x})\right]=\boldsymbol{P}^{\boldsymbol{T}}\left(\boldsymbol{P}^{\boldsymbol{T}} \boldsymbol{W} \boldsymbol{P}+\boldsymbol{H}\right)^{-\mathbf{1}} \boldsymbol{P}^{T} \boldsymbol{W}$

\section{Numerical examples and algorithm verification}

We verify the accuracy of the proposed meshless scheme through several flow cases. First, we considered the two most frequently used benchmark problems in Computational Fluid Dynamics (CFD), the lid-driven cavity flow and the backward-facing step (BFS). Next, we examined flow cases in irregular geometries, starting with a 2D rectangular duct with a cylinder (and multiple cylinders) as an obstacle and a geometry that resembles flow in a blood vessel. Finally, we consider the 3D lid-driven cavity flow problem as well as a typical 3D geometry of a dilated vessel (aneurysm), obtained by segmenting CT (computed tomography) images. For the test cases presented we considered Reynolds numbers that correspond to steady state solutions. In detail, for the lid-driven cavity flow problem steady state solutions can be obtained for Reynolds number up to $R e=10,000$; for the rest of the flow cases we considered moderate Reynolds numbers to ensure steady state solutions. Additionally, moderate Reynolds numbers ensure, for the flow cases considered in the present study, laminar flow; this is compatible with the flow equations being solved,whichare only valid for laminar incompressible flows.

For the simulations conducted we used two types of nodal configurations: $(i)$ a uniform semi-staggered grid (Type-I) embedded in the geometry and (ii) a semi-staggered grid (Type-II) based on a triangular (or tetrahedral in 3D) mesh of the spatial domain. The vertices of the nodes correspond to the velocity nodes, while the barycenter of triangles to 
the pressure nodes (this configuration resembles the mini-elements configuration used in FEM). Figure 1 shows the nodal configurations used for the case of an irregular geometry. It is worth mentioning that results were also computed for collocated nodal distribution, where velocity and pressure values are computed on the same nodes.

\subsection{Lid-driven cavity problem}

The flow in closed cavities, mechanically driven by tangentially moving walls, is a wellknown benchmark problem for viscous incompressible flow. The forced convection in a square cavity with isothermal sides and moving upper side is known as the lid-driven cavity problem. The lid-driven cavity flow problem, despite its geometrical simplicity, is considered to be a challenging problem, mainly due to the vortices that appearat the corners of the square cavity, especially for high Reynolds numbers. The flow boundary conditions imposed were no-slip boundary condition forall walls of the cavity, except for the top wall whichmoveswith velocity $u=1$ in the $x$-direction.

For all the simulations conducted we consider both the uniform and irregular nodal distributions previously described. For the construction of the shape functions and derivatives, in the case of uniform nodal distribution, the support domain was defined by using the 20 nearest neighbors, while in the case of irregular nodal distribution, an adaptive cut-off radius has been used, adapting accordingly the number of nodes in the support domain. Coarse and fine nodal configurations were used in order to obtain a gridindependent solution.

In the case of uniform nodal distribution (Type-I), a uniform grid of $121 \times 121$ nodes (for velocity components) was used for $R e=100$, while for $R e=400$ and $R e=1,000$ a $241 \times 241$ grid was used. Nevertheless, nodal distributions as coarse as $41 \times 41$ and $81 \times 81$ were adequate for the convergence and accuracy of the numerical solution. Additionally, computations were conductedfor higher values of the Reynolds number up to $R e=10,000$, using a uniform nodal distribution of $401 \times 401$ nodes. The results obtainedwere compared against the values provided in Ghia et al. [40] at the centerlines ( $x=0.5$ and $y=0.5$ ), with the convergence curve of $L_{\text {inf }}$ (the maximum absolute value of the difference between the two solutions) and $L_{2}$ (the mean square value of the difference 
between the two solutions) shown in Fig. 2. We assess the accuracy of the method for $R e=400,1,000,5,000$ and 10,000 through qualitative and quantitative comparisons with benchmark numerical results from the literature [40, 41]. For qualitative evaluation of the solution, pressure contours (Fig. 3) and streamlines (Fig. 4, computed as in [41]) of the flow field are presented for $R e=400,1,000,5,000$ and 10,000. The streamlines plotted in Fig. 4show the formation of the counter-rotating secondary vortices that appear as the Reynolds number increases. For quantitative comparison, we plot the $u$-velocity profiles along a vertical line and the $v$-velocity profiles along a horizontal line passing through the geometric center of the cavity, respectively, in Fig. 5, for $R e=400,1,000,5,000$ and 10,000. These profiles are in good agreement with of the results from Ghia et al. [40], shown by symbols in Fig. 5.

In the case of irregular nodal distribution (Type-II), triangle vertices were used as the nodal distribution where velocity values are calculated. Pressure values were calculated at the barycenter of each triangular element. For the case of $R e=100$ we started using a coarse grid consisting of 2,601 velocity nodes and 5,000 pressure nodes and gradually refined the grid. The IPOT-MMLS scheme converges to the data obtained for the benchmark problem.

For every type of nodal distribution, as the number of nodes in the support domain increases, the accuracy of the proposed scheme increases.

\subsection{Backward facing step (BFS) problem}

The second benchmark problem considered is the backward-facing step (BFS) flow problem [42]. The flow domain is a channel of width $H(H=1)$ and length $L(L=30 H)$, with an obstacle (step) (of height $H=0.5$ ) placed at the left-most edge of the inlet $(x=0)$. Flow is assumed to be fully developed, with a parabolic inflow velocity profile given by $\boldsymbol{u}=(12 y$ $24 y^{2}, 0$ ) for $y>0.5$. Fully developed flow is also assumed at the outlet (right edge), with the velocity profile given by $\boldsymbol{u}=\left(0.75-3 y^{2}, 0\right)$ for $0<y<1$. At the bottom and top walls no-slip boundary conditions were imposed.

BFS is considered as a demanding benchmark flow problem due to the vortices formed downstream, just after the step. The BFS has been studied both experimentally [42] and numerically [42-45]. The flow has been found to be stable and two-dimensional for 
Reynolds number $R e<400$, allowing the flow to be numerically modeled in $2 \mathrm{D}$ and compared directly with experiments [42]. Beyond this Reynolds number, the flow is 3D and the $2 \mathrm{D}$ approximation is no longer valid. However, numerical results for the $2 \mathrm{D}$ problem for $\operatorname{Re}>400$ are still given in the literature as a purely numerical benchmark problem [43]. Here we present numerical results for our method for $R e=200$, for comparison with both experimental and numerical results such as [42].

Initially we tested the performance of the proposed scheme using the Type-I nodal distribution. To ensure a grid-independent numerical solution, successively increasing nodal configurations were tested starting from $300 \times 11,600 \times 21,900 \times 31$ and $1200 \times 41$. For the $R e=200$, a uniform nodal distribution of $27,931(900 \times 31)$ is used. Fig. 6 shows the streamlines and vorticity contours for $R e=200$. We can observe that the flow separates at the step corner and a vortex is formed downstream. For $R e=200$ the reattachment length of the formed vortex is $L_{\text {reattachment }}=2.55$, being in agreement with that computed using different numerical methods, such as Meshless Collocation Point with Radial Basis Functions and Finite Element Method [25]. Type-II nodal configurations were also used, starting from a coarse distribution and resulting in a denser one after successive refinements (total number of nodes used were 12,321). The numerical results obtained showed excellent agreement with those of Type-I. For the case of $R e=200$ the reattachment length computed was $L_{\text {reattachment }}=2.54$.

\subsection{Flow behind cylinder}

In the next example we consider the laminar flow in a rectangular duct with a cylindrical obstacle. The duct has length $L=2.2 \mathrm{~m}$ and height $H=0.41 \mathrm{~m}$. The cylindrical obstacle is located at point $\boldsymbol{O}(0.2,0.2)$ having radius $r=0.05 \mathrm{~m}$. The kinematic viscosity of the fluid was set to $v=0.001 \mathrm{~m}^{2} / \mathrm{s}$. The Reynolds number is defined by $R e=2 u r / v$ with the mean velocity $u_{\text {mean }}=(2 / 3) * u(0, H / 2)$. The inflow conditions are

$$
(u(\mathrm{y}), v)=\left(4 u_{m} y\left(\frac{H-y}{H^{2}}\right), 0\right)
$$


with $u_{m}=0.3 \mathrm{~m} / \mathrm{s}$ yielding Reynolds number $R e=20$. At the outlet a fully developed flow $(\partial u / \partial x=0)$ is applied, while at the rest of the boundaries no-slip conditions were applied. Simulations were conducted using the different types of nodal configuration described above. The numerical results obtained were compared point wise with those computed using COMSOL [46]. The maximum absolute values of the difference between the two solutions $L_{i n f}$, for the velocity components $u$ - and $v$-, were computed as $1.14 \times 10^{-3}$ and $1.82 \times 10^{-3}$, respectively. The uniform semi-staggered nodal distribution (Type-I) was more stable in terms of computing shape functions and derivatives. The positivity conditions [47] were fulfilled naturally, in contrast to the Type-II configuration, where extra care should be taken in order to find the optimal support domain.

For the results obtained using the uniform semi-staggered distribution, the contour plots of the velocity components are shown in Fig. 7, and pressure contours in Fig. 8. We used 9,546 nodes with 90 of them distributed over the cylinder circumference. Finally, Fig. 9 shows $u$ - velocity profile along the line $x=0.25$ and $x=0.3$ for $R e=20$ comparing the results of IPOT-MMLS technique and COMSOL. All flow variables are computed in SI units. Contour plots of the velocity components and pressure are presented for Reynolds number up to $\operatorname{Re}=60\left(u_{m}=0.9 \mathrm{~m} / \mathrm{s}\right)$. For higher values of maximum inlet velocity $\boldsymbol{u}_{m}$, and, consequently for higher values of $R e$, the flow regime becomes oscillating downstream and is not considered as steady state flow.

Additionally, we consider the case where a number of cylinders are present downstream, using the exact same boundary conditions as in the case of a single cylinder. In total 18,362 nodes were used, 630 of them located on the boundaries. The cut-off radius was chosen such that 40 nodes were located in the support domain. The numerical results obtained were compared against those computed using COMSOL, with the maximum absolute values of the difference between the two solutions for the velocity components $u$ - and $v$ computed as $1.2 \times 10^{-3}$ and $2.3 \times 10^{-3}$, respectively. The contour plots for the velocity components are shown in Fig. 10, while pressure contours are plotted in Fig. 11. Figure 12 shows $u$ - velocity profile along the line $x=0.25$ and $x=0.3$ for $R e=20$ comparing the results of IPOT-MMLS technique and COMSOL, whenusinga staggered grid based on irregular nodal distribution obtained from a triangular mesh. The 40 closest nodes were used to define the support domain of each node. 


\subsection{Bypass geometry}

As a fourthexample, we consider the flow in an irregular geometry that resembles a 2D stenosed artery with a bypass, Fig. 13, having length of $15 \mathrm{~cm}$. For the simulations conducted, we considered a parabolic velocity profile with $u=4 u_{m}\left(y-y^{2}\right)$ and $v=0$ in the inlet of the domain, at the outlet fully developed flow, and on the remaining walls no-slip boundary conditions $(u=v=0)$. For $u_{m}=0.1 \mathrm{~m} / \mathrm{s}$ and kinematic viscosity of $\boldsymbol{v}=\mathbf{0 . 0 0 1} \boldsymbol{m}^{2} / \mathbf{s}$ the Reynolds number was calculated as $R e=40$, giving rise to laminar flow regime. The proposed scheme can provide accurate results for higher Reynolds numbers; in the case of $v=0.0001 \mathrm{~m}^{2} / \mathrm{s}$ Reynolds number becomes $R e=240$. For higher $R e$ numbers turbulence may probably occur. Since studying turbulence is out of the scope of the present study, we restricted the presented results to low and moderate Reynolds numbers.

Both nodal configuration types were used in order to test the applicability of the proposed scheme in flow cases concerning irregular domains. All the numerical solutions, obtained using different nodal distribution types, converged. The numerical results computed are accurate as compared to the results obtained using the finite element method (COMSOL). The maximum absolute values of the difference between the two solutionsfor the velocity components $u$ - and $v$ - were computed as $2.4 \times 10^{-3}$ and $3.6 \times 10^{-3}$, respectively. Simulations show that the most reliable, in terms of computational cost, stability and convergence, has been the uniform semi-staggered embedded distribution. Several grids with increasing resolution were used in order to obtain a grid independent solution. For the simulations conducted we used a total of 14,563 nodes, with 1,380 of them located on the boundaries. Figure 14 shows iso-contours for the velocity components $u, v$, pressure and streamlines. All flow variables are computed in SI units.

\subsection{Lid-driven flow in $3 D$}

The lid-driven flow in a cubic cavity $(0,1)^{3}$ is the natural extension to $3 \mathrm{D}$ of the $2 \mathrm{D}$ driven cavity test case, Fig. 15 . Numerical results were obtained for $R e=100$ and $R e=400$. 
A grid independence study has been conducted to ensure a grid independent numerical solution. Typical nodal distributions up to $41 \times 41 \times 41(68,000)$ and $61 \times 61 \times 61(226,981)$ points for $R e=100$ and 400, respectively, are employed. The numerical results obtained are in a good agreement with those in [48], Table 1. Velocity profile at the mid-planes for $R e=100$ and 400, using successively denser nodal distributions, are shown in Fig. 16.

\subsection{Flow in 3D aneurysm}

We considered the flow in a 3D geometry obtained using a CT scan showing the part of the blood vessel that suffers from an aneurysm, Fig. 17. The aneurysmatic part of the vessel has been smoothed during the segmentation and 3D reconstruction procedure, and the final output was an STL file with all the geometrical information. The length of the vessel is $L=10 \mathrm{~cm}$ and the radius is $R=1 \mathrm{~cm}$. Concerning the boundary conditions imposed, at the inlet a plug flow velocity profile $(u=1 \mathrm{~cm} / \mathrm{s}, v=w=0)$, and at the outlet a zero axial velocity gradient was imposed $(\partial u / \partial x=0)$. On the rest of the boundaries no-slip conditions were applied. The 3D mesh, consisting of tetrahedral elements, has been created in ANSYS ICEM, the pre-processor contained in ANSYS CFX commercial software [49].

For the simulations conducted the STL file used, shown in Fig. 18a, describes the surface of the vessel geometry, which is represented by triangles and forming a convex surface. All types of nodal configurations described above were used in the simulations conducted. In the case of the uniform grid embedded in the geometry, a grid-independent solutionwas obtained by successively increasing the number of nodes. The numerical solution was compared against the numerical solution given by ANSYS CFX. For the simulations conducted, the distance $h$ of the support domain was set to $h=0.2 \mathrm{~cm}$ (30 nodes in the support domain), with a total of 12,456 nodes used inthe computations.By comparing theIPOT/MMLS results with those computed by ANSYS CFX, the maximum absolute values of the difference between the two solutions were $1.2 \times 10^{-3}, 2.3 \times 10^{-3}$ and $3.6 \times 10^{-3}$ for $u$-, $v$ - and $w$ - velocity components, respectively.

In order to depict the direct applicability of the proposed scheme in cases where the nodal distributions are directly given using a tetrahedral mesh, we considered a Type-II nodal distribution, with the nodes of the tetrahedral mesh are used for the computation of 
the velocity field values. The pressure field values were computed at the barycenter of the tetrahedral elements. The velocity nodes are shown in Fig. 18b and the pressure nodes in Fig. 18c. The pressure contours on the surface of the vessel are shown in Fig. 19a, while streamlines are plotted in Fig. 19b (in a view angle suitable for streamlines in 3D). All flow variables are computed in SI units.

\section{Conclusions}

In this paper we applied the Modified Moving Least Squares (MMLS) meshless method in the numerical solution of laminar, incompressible, steady state flow in the context of a point collocation method. We solved the flow governing equations in their primitive variables (velocity-pressure) formulation, by using a semi-staggered meshless nodal distribution. A pressure correction methodology (IPOT method) has been used in order to fulfil the incompressibility constraint, ensuring the mass conservation. The advantages of the proposed IPOT meshless point collocation are that it is straightforward and easy to apply, results can be obtained using collocated or semi-staggered nodal distributions, it works for 2D and 3D geometries, it is truly meshless, while there is no need for pressure boundary conditions since no pressure constitutive equation is solved. Additionally, in our approach there is noneed for the computation of the velocity potential or unit normal vectors. The proposed scheme can be used for the numerical solution of the transient Navier-Stokes equations; the iterative procedure, used to update the solution in the steady state equations, can be used to update in time the numerical solution when an explicit or an implicit solver is used. Additionally, the method is useful for the flow cases where pressure boundary conditions cannot be defined. The absence of pressure boundary conditions can be handledby the IPOT scheme. In case where the pressure on the boundaries is known the IPOT method can also be applied. Nevertheless, for the last case more numerical studies are needed.

The MMLS approximation method makes use of two distinct sets of nodes for velocity and pressure variables. We have focused on the method's performance, accuracy and stability using uniformly and irregularly distributed nodes. First, we showed that the proposed scheme provides accurate results in uniform geometries using benchmark fluid 
flow problems, namely the lid-driven cavity and the backward-facing step. Both qualitative and quantitative results were given in order to show the accuracy of the method used. Second, we tested the numerical stability and performance of the method across complexgeometry problems. COMSOL and ANSYS CFX were used as reference solvers for the complex geometries, demonstrating that the proposed scheme provides consistent results across all the problems considered. Concerning the parameters related to upwinding for moderate Reynolds numbers, in both COMSOL and ANSYS CFX, the default parameters have been used in all the test cases considered. MMLS numerical scheme was stable with both uniform and irregular nodal distributions.

Our results show that the use of MMLS operators in conjunction with velocity and pressure correction method provide stable and accurate solutions across a range of $2 \mathrm{D}$ and $3 \mathrm{D}$ problems, in both regular and irregular geometries. For every type of nodal distribution, as the number of nodes in the support domain increases, the accuracy of the proposed scheme increases. The same happens when the total number of nodes in the spatial domain is increased, where uniform nodal distributions embedded in geometry seem to be more efficient. Finally, for future applications, the proposed scheme will be upgraded to treat time dependent flow problems, pressure-driven and shear-driven flow, considering flow regimes with high Reynolds number.

Acknowledgments: The financial support of Australian Research Council (Discovery Grant no. DP160100714) is gratefully acknowledged. We wish to acknowledge the Raine Medical Research Foundation for funding G. R. Joldes through a Raine Priming Grant. 


\section{References}

1. Orszag SA, Israeli M, Deville MO. Boundary conditions for incompressible flows. J Scient Comput 1986; 1:175-111.

2. Ferziger JH, Peric M. Computational Methods for Fluid Dynamics. Springer; 2002.

3. Harlow FH, Welch JE. Numerical calculation of time-dependent viscous incompressible flow of fluid with free surface. Phys Fluids 1965; 8:2182-2189.

4. Chorin AJ. Numerical solution of the Navier-Stokes equations. Math Comp 1968; 22:745-762. 
5. de Vries G, Norrie DH. The application of the finite-element technique to potential flow problems. J Appl Mech, ASME Transactions 1971; 38:798-802.

6. Zienkiewicz OC, Taylor RL, Nithiarasu P. The Finite Element Method for Fluid Dynamics. Sixth edition, Elsevier: Butterworth-Heinemann; 2005.

7. Gresho PM, Sani RL (1998) Incompressible Flow and the Finite Element Method. Wiley.

8. Patankar SV, Spalding DB. A calculation procedure for heat, mass and momentum transfer in three-dimensional parabolic flows. Int J Heat Mass Transfer 1972; 15:17871806.

9. Patankar SV. Numerical Heat Transfer and Fluid Flow. McGraw-Hill: New York; 1980.

10. Chorin AJ. A numerical method for solving incompressible viscous flow problems. J Comput Phys 1967; 2:21-26.

11. Papadopoulos $P K$, Hatzikonstantinou PM. Improved CVP scheme for laminar incompressible flows. Int J Numer Meth Fluids 2011; 65:1115-1132.

12. Hafez M, Shatalov A, Wahba E. Numerical simulations of incompressible aerodynamic flows using viscous/inviscid interaction procedures. Comput Meth Appl Mech Engineer 2006; 195:3110-3127.

13. Papadopoulos PK. An auxiliary potential velocity method for incompressible viscous flow. Computers \& Fluids 2011; 51:60-67.

14. Papadopoulos PK. An implicit potential method for incompressible flows. Int J Numer Meth Engineer 2013; 94:672-686. 
15. Nguyena VPh, Rabczuk T, Bordas S, Duflot M. Meshless methods: A review and computer implementation aspects. Mathem Comput Simul 2008; 79:763-813.

16. Lin H, Atluri SN. The meshless local Petrov-Galerkin (MLPG) method for solving incompressible Navier-Stokes equations. Comput Model Eng Sci 2001; 2:117-142.

17. Mohammadi MH. Stabilized Meshless Local Petrov-Galerkin (MLPG) Method for Incompressible Viscous Fluid Flows. Comput Model Eng Sci 2008; 29:75-94.

18. Arefmanesh A, Najafi M, Abdi H. Meshless Local Petrov-Galerkin Method with Unity Test Function for Non-Isothermal Fluid Flow. Comput Model Eng Sci 2008; 25:9-22.

19. Loukopoulos VC, Bourantas GC. MLPG6 for the solution of incompressible flow equations. Comput Model Eng Sci 2012; 88:531-558.

20. Baez E, Nicolas A. Recirculation of viscous incompressible flows in enclosures. Comput Model Eng Sci 2009; 41:107-130.

21. Grimaldi A, Pascazio G, Napolitano M. A Parallel Multi-block Method for the Unsteady Vorticity-velocity Equations. Comput Model Eng Sci 2006; 14:45-56.

22. Sellountos EJ, Sequeira A. An advanced meshless LBIE/RBF method for solving twodimensional incompressible fluid flows. Comput Mech 2008; 41:617-631.

23. Sellountos EJ, Sequeira A. Hybrid Multi-region BEM/LBEI-RBF Velocity-Vorticity Scheme for the Two-Dimensional Navier-Stokes Equations. Comput Model Eng Sci. 2008; 23:127-147.

24. Bourantas GC, Skouras ED, Loukopoulos VC, Nikiforidis GC. Meshfree point collocation schemes for 2D steady state incompressible Navier-Stokes equations in 
velocity-vorticity formulation for high values of Reynolds number. Comput Model Eng Sci 2010; 59:1-33.

25. Bourantas GC, Skouras ED, Loukopoulos VC, Nikiforidis GC. Numerical solution of non-isothermal fluid flows using local radial basis functions (LRBF) interpolation and a velocity-correction method. Comput Model Eng Sci 2010; 64:187-212.

26. Bourantas GC, Loukopoulos VC. A meshless scheme for incompressible fluid flow using a velocity-pressure correction method. Computers \& Fluids 2013; 88:189-199.

27. Kosec G, Šarler B. Solution of thermo-fluid problems by collocation with local pressure correction, Int J Numer Meth Heat Fluid Flow 2008; 18:868-882.

28. Mai-Duy N, Mai-Cao L, Tran-Cong T. Computation of transient viscous flows using indirect radial basic function networks. Comput Model Eng Sci 2007; 18:59-77.

29. Zhang L, Ouyang J, Zhang XH. On a two-level element-free Galerkin method for incompressible fluid flow. Appl Numer Math 2009; 59:1894-1904.

30. Huerta A, Vidal Y, Villon P. Pseudo-divergence-free element free Galerkin method for incompressible fluid flow. Comput Methods Appl Mech Engrg 2004; 193:1119-1136.

31. Shadloo MS, Zainali A, Sadek SH, Yildiz M. Improved Incompressible Smoothed Particle Hydrodynamics method for simulating flow around bluff bodies. Comput Methods Appl Mech Engrg 2011; 200:1008-1020.

32. Napoli E, De Marchis M, Vitanza E. PANORMUS-SPH. A new Smoothed Particle Hydrodynamics solver for incompressible flows. Computers \& Fluids 2015; 106:185-195.

33. Ellero M, Serrano M, Espanol P. Incompressible smoothed particle hydrodynamics. J Comput Phys 2007; 226:1731-1752. 
34. Oñate E, Idelsohn S, Zienkiewicz OC, Taylor RL, Sacco C. A stabilized finite point method for analysis of fluid mechanics problems. Comput Methods Appl MechEngrg 1996;139:315-346.

35. Tiwari S, Kuhnert J. Finite Pointset Method Based on the Projection Method for Simulations of the Incompressible Navier-Stokes Equations. Lect Notes Comput Sci Eng $2003 ; 26: 373-388$.

36. Tiwari S, Kuhnert J. Modeling of two-phase flows with surface tension by finite pointset method (FPM). J Comput Appl Math 2007; 203:376-386.

37. Lancaster P, Salkauskas K. Surfaces generated by moving least squares method. Math Comput 1981; 37:141-158.

38. Joldes GR, Chowdhuty HA, Wittek A, Doyle B, Miller K. Modified moving least squares with polynomial bases for scattered data approximation. Appl Math Comput 2015; 266:893-902.

39. Chowdhury H, Joldes G, Wittek A, Doyle B, Pasternak E, Miller K. Implementation of a Modified Moving Least Squares Approximation for Predicting Soft Tissue Deformation Using a Meshless Method. In: Doyle B, Miller K, Wittek A, Nielsen PMF, editors. Computational Biomechanics for Medicine: Springer International Publishing 2015; p. 5971.

40. Ghia U, Ghia KN, Shin CT. High-Re solutions for incompressible flow using the Navier-Stokes equations and a multigrid method. J Comput Phys 1982;48:387-411.

41. Erturk E, Corke TC, Gokcol C. Numerical solutions of 2-D steady incompressible driven cavity flow at high Reynolds numbers. Int J Numer Methods Fluids 2005; 48:747774. 
42. Armaly BF, Durst F, Pereira JCF, Schonung B. Experimental and theoretical investigation of backward-facing step flow. J Fluid Mech 1983; 172:473-496.

43. Gartling DK. A test problem for outflow boundary conditions - flow over a backwardfacing step. Int J Numer Meth Fluids 1990; 11:953-967.

44. Kim J, Moin P. Application of a fractional-step method to incompressible NavierStokes equations. J Comput Phys 1985;59:308-323.

45. Sohn J. Evaluation of fidap on some classical laminar and turbulent benchmarks. Int J Numer Meth Fluids 1988; 8:1469-1490.

46. COMSOL Multiphysics product.

47. Jin X, Li G, Aluru NR. Positivity conditions in meshless collocation methods. Comput Methods Appl Mech Engrg 2004; 193:1171-1202.

48. Ding $\mathrm{H}$, Shu $\mathrm{C}$, Yeo KS, Xu D. Numerical computation of three-dimensional incompressible viscous flows in the primitive variable form by local multiquadric differential quadrature method. Comput Methods Appl Mech Engrg 2006; 195:516-533.

49. ANSYS ${ }^{\circledR}$ Academic Research. 


\section{Table captions}

Table 1. Comparison of numerical results for lid-driven flow in a cubic cavity. $u$ component of the velocity profile at the mid-planes for $R e=100$. 


\section{Figure captions}

Fig. 1. Nodal configurations used for the solution of the governing equations $(i)$ uniform semi-staggered grid (velocity is defined on the blue dots and pressure on the red ones. On the boundary there are velocity nodes) and (ii) irregular semi-staggered grid.

Fig. 2. Lid-driven cavity flow: Convergence for $R e=400$ for increasing resolution: $81 \times 81$, $121 \times 121,161 \times 161$ and $201 \times 201$. The absolute differences between the two solutions $L_{i n f}$ and $L_{2}$ over all nodes are calculated with respect to a solution computed with $261 \times 261$ nodes, as in [40]. $N \times N$ is the total number of nodes used in the simulation, and $N$ the number of nodes in each direction.

Fig. 3. Lid-driven cavity flow: Pressure contours for (a) $R e=400$ (b) $R e=1,000$ (c) $R e=5,000$ and (d) $R e=10,000$.

Fig. 4. Lid-driven cavity flow: Streamline patterns of primary and secondary vortices (computed as in [41]) obtained for (a) $R e=400$ (b) $R e=1,000$ (c) $R e=5,000$ and (d) $R e=10,000$. The full domain, close-up on the left eddy and close-up on right eddy are defined as in [41].

Fig. 5. The $u$-velocity on the section $x=0.5$ and the $v$-velocity on the section $y=0.5$ of the square lid-driven cavity problem for (a) $R e=400$ (b) $R e=1,000$ (c) $R e=5,000$ and (d) $R e=10,000$. Results of Ghia et al. [40] are compared with the current numerical solutions.

Fig.6. (a) Pressure and (b) streamlines contours for the backward-facing step for $R e=200$.

Fig. 7. Velocity contours for $u$ - component and $v$-component, respectively for Reynolds number (a) $R e=20$ and (b) $R e=60$.

Fig. 8. Pressure contours for (a) $R e=20$ and (b) $R e=60$. 
Fig. 9. $u$ - velocity components profile along the line $x=0.25$ and $x=0.3$ for $R e=20$. Solid line is for IPOT-MMLS scheme and squre symbol for COMSOL.

Fig. 10. Velocity contours for (a) $u$ - component and (b) $v$ - component, for $R e=20$.

Fig. 11. Pressure contours for $R e=20$.

Fig. 12. $u$-velocity components profile along the line $x=0.65$ and $x=0.95$ for $R e=20$. Solid line is for IPOT-MMLS scheme and squre symbol for COMSOL.

Fig. 13. Irregular geometry that resembles a $2 \mathrm{D}$ stenosed artery with a bypass.

Fig. 14. For the bypass geometry (a) pressure contours (b) $u$-velocity component contours (c) $v$ - velocity component contours(d) streamlines for $R e=20$ and (e) pressure contours (f) $u$ - velocity component contours (g) $v$ - velocity component contours (h) streamlines for $R e=240$.

Fig. 15. Geometry of the lid-driven flow in a cubic cavity.

Fig. 16. Velocity component distribution along the vertical centerline of cubic cavity, $u-y$ for (a) $R e=100$ and (b) $R e=400$ using successively denser nodal distributions. Square symbol is for the results in [48].

Fig. 17. 3D geometry obtained using a CT scan showing the part of the blood vessel that suffers from an aneurysm.

Fig. 18. (a) Surface triangulation of the vessel geometry and nodal distribution for (b) velocity (c) pressure field values.

Fig. 19. (a) Pressure contours on the vessel wall and (b) streamlines (in a view angle suitable for streamlines in 3D) for $R e=80$. 
Table 1

\begin{tabular}{ccccc}
\hline $\boldsymbol{y}$ & Ding $\boldsymbol{e t} \boldsymbol{a l} . \mathbf{[ 4 8}]$ & IPOT-MMLS & error & Relative error (\%) \\
\hline 0.00 & 0 & 0 & 0 & \\
0.04 & -0.028 & -0.025 & 0.003 & 10.7 \\
0.08 & -0.052 & -0.047 & 0.005 & 9.61 \\
0.12 & -0.073 & -0.066 & 0.007 & 9.59 \\
0.16 & -0.092 & -0.084 & 0.008 & 8.69 \\
0.20 & -0.112 & -0.101 & 0.011 & 9.82 \\
0.24 & -0.130 & -0.118 & 0.012 & 9.23 \\
0.28 & -0.148 & -0.135 & 0.013 & 8.78 \\
0.32 & -0.166 & -0.152 & 0.014 & 8.43 \\
0.36 & -0.181 & -0.167 & 0.014 & 7.73 \\
0.40 & -0.194 & -0.180 & 0.014 & 7.22 \\
0.44 & -0.202 & -0.190 & 0.012 & 5.94 \\
0.48 & -0.205 & -0.196 & 0.009 & 4.39 \\
0.52 & -0.202 & -0.197 & 0.005 & 2.47 \\
0.56 & -0.191 & -0.190 & 0.001 & 0.52 \\
0.60 & -0.173 & -0.175 & 0.002 & 1.15 \\
0.64 & -0.147 & -0.152 & 0.005 & 3.40 \\
0.68 & -0.113 & -0.118 & 0.005 & 4.42 \\
0.72 & -0.071 & -0.075 & 0.004 & 5.63 \\
0.76 & -0.019 & -0.018 & 0.001 & 5.26 \\
0.80 & 0.048 & 0.052 & 0.004 & 8.33 \\
0.84 & 0.140 & 0.151 & 0.011 & 7.86 \\
0.88 & 0.270 & 0.283 & 0.013 & 4.81 \\
0.92 & 0.456 & 0.465 & 0.009 & 1.97 \\
0.96 & 0.707 & 0.720 & 0.013 & 1.84 \\
1.00 & 1.000 & 1.000 & 0 & \\
\hline
\end{tabular}




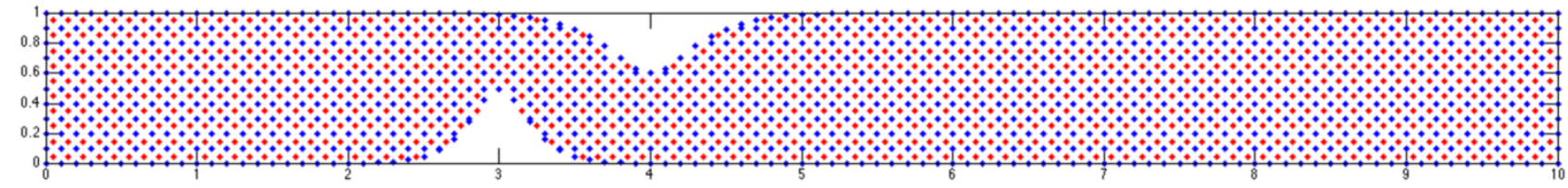

(a)
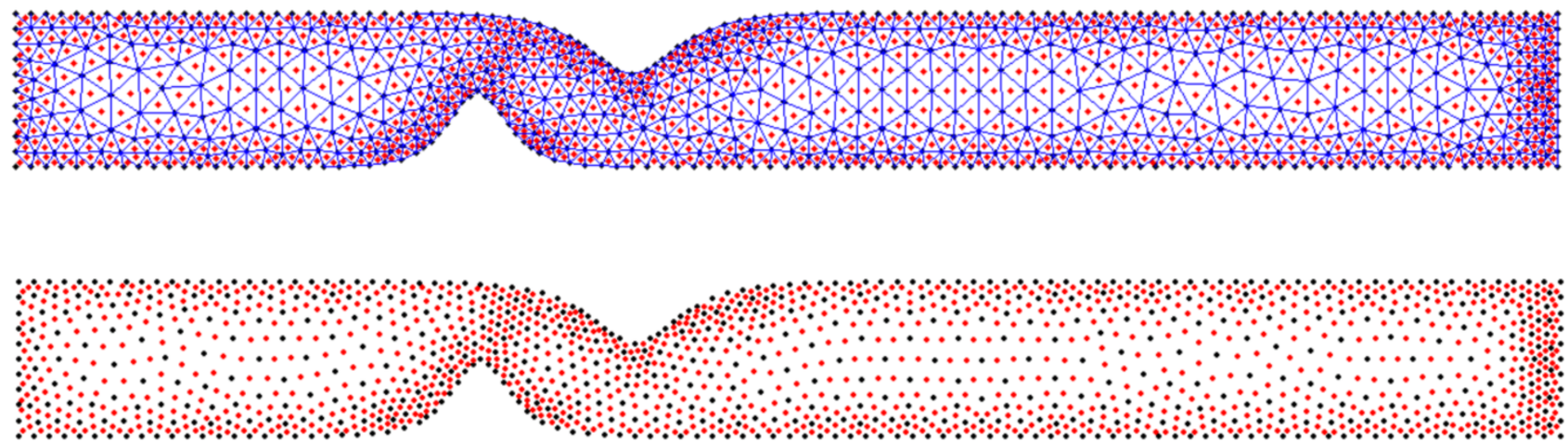

(b)

Fig. 1 


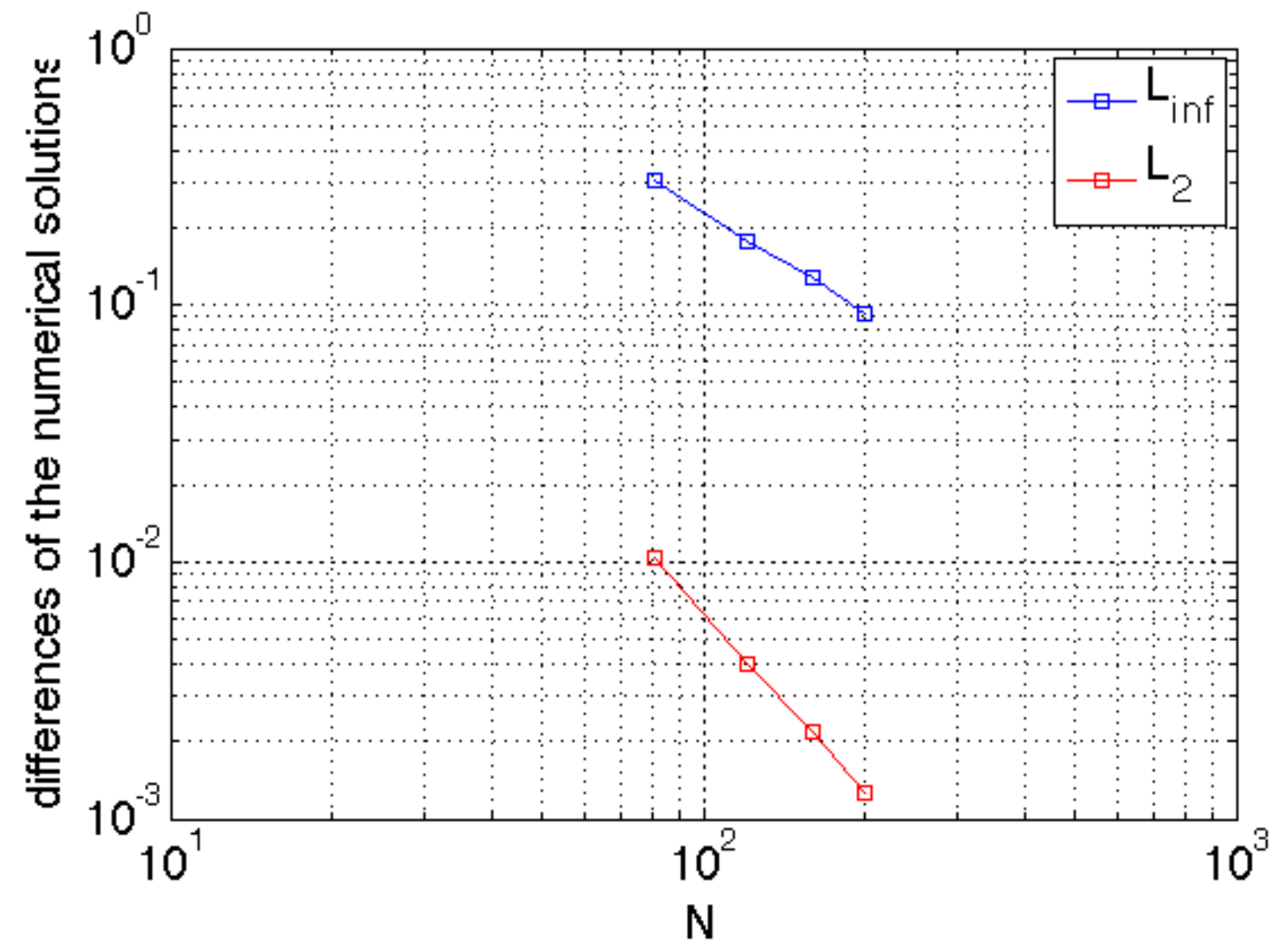

Fig. 2 


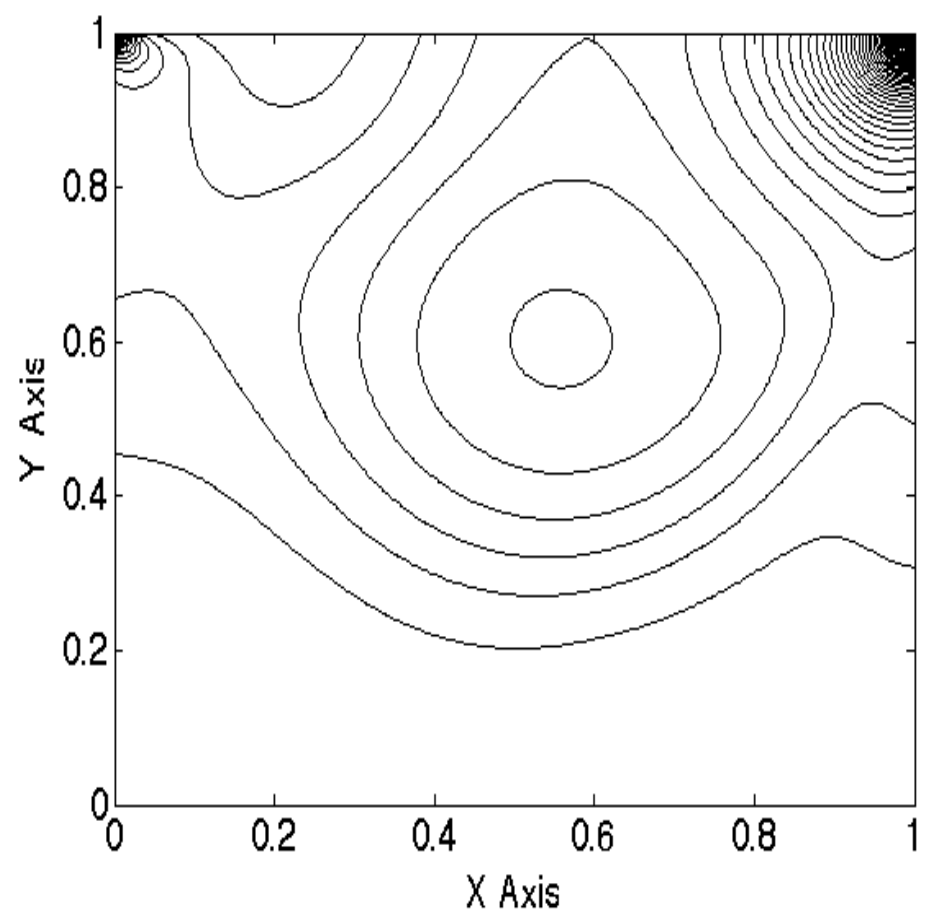

(a)

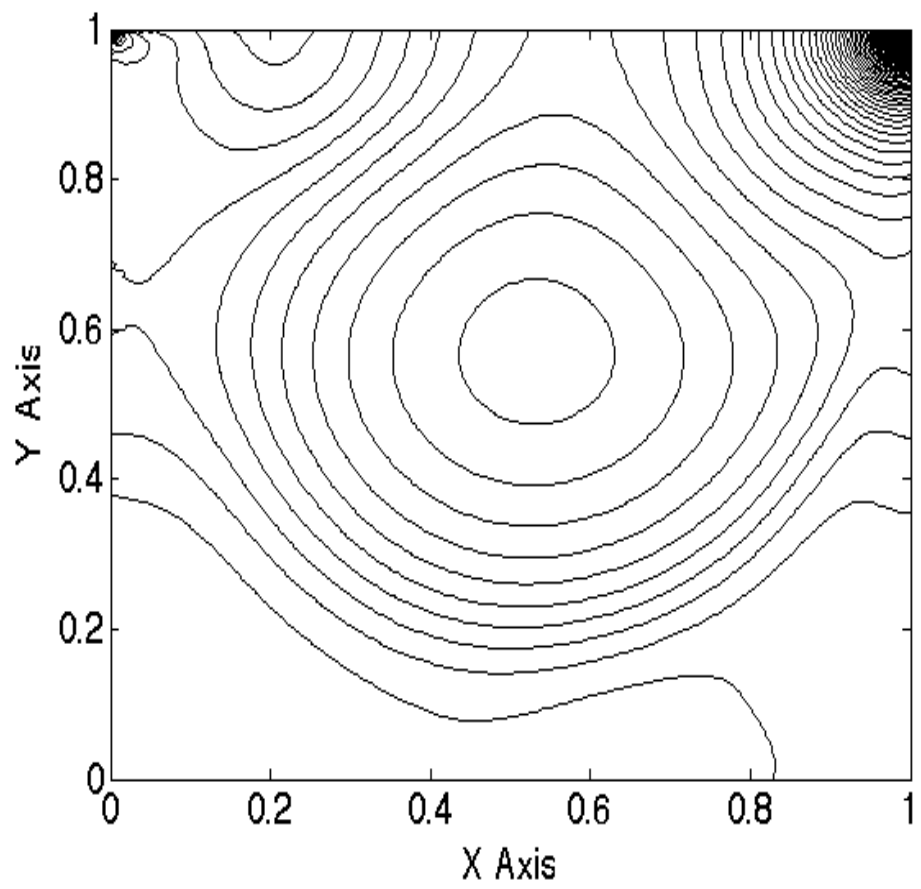

(b) 


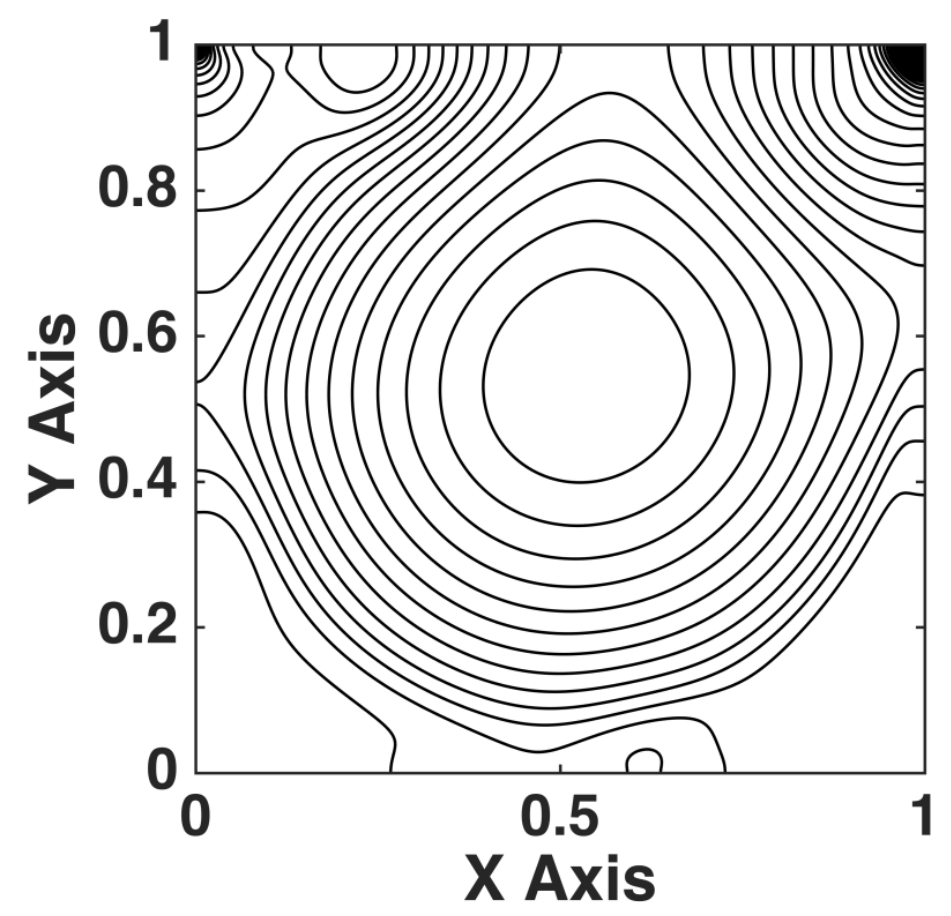

(c)

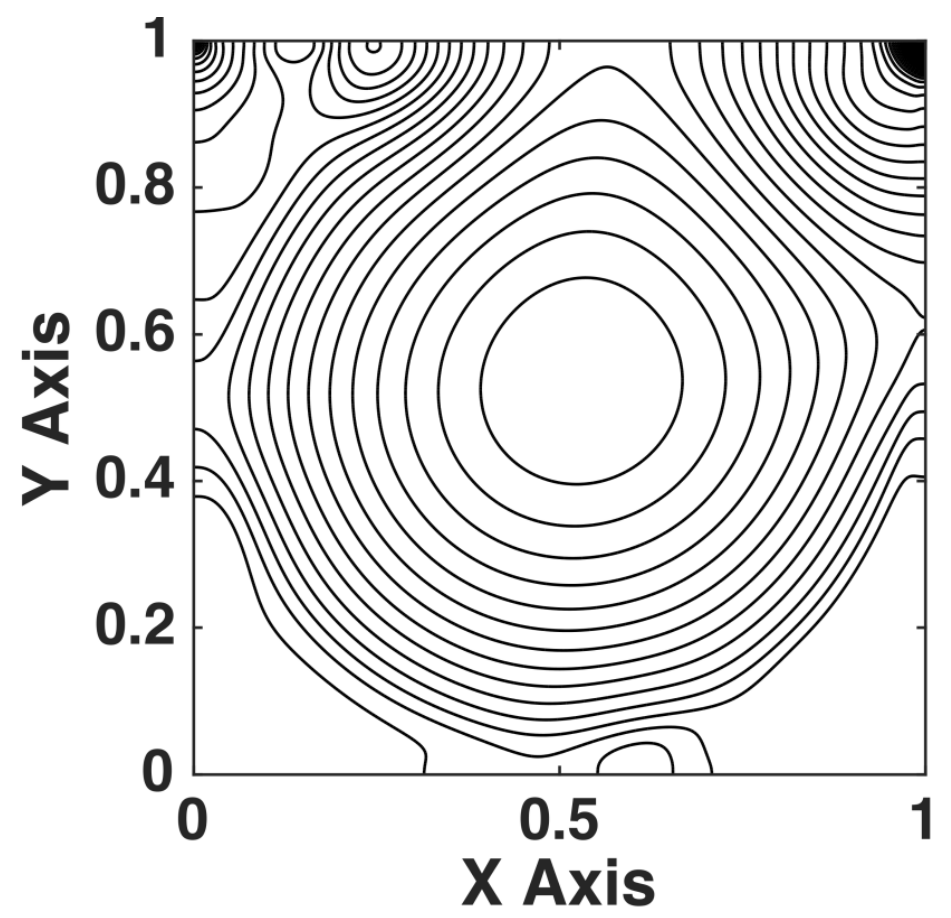

(d)

Fig. 3 

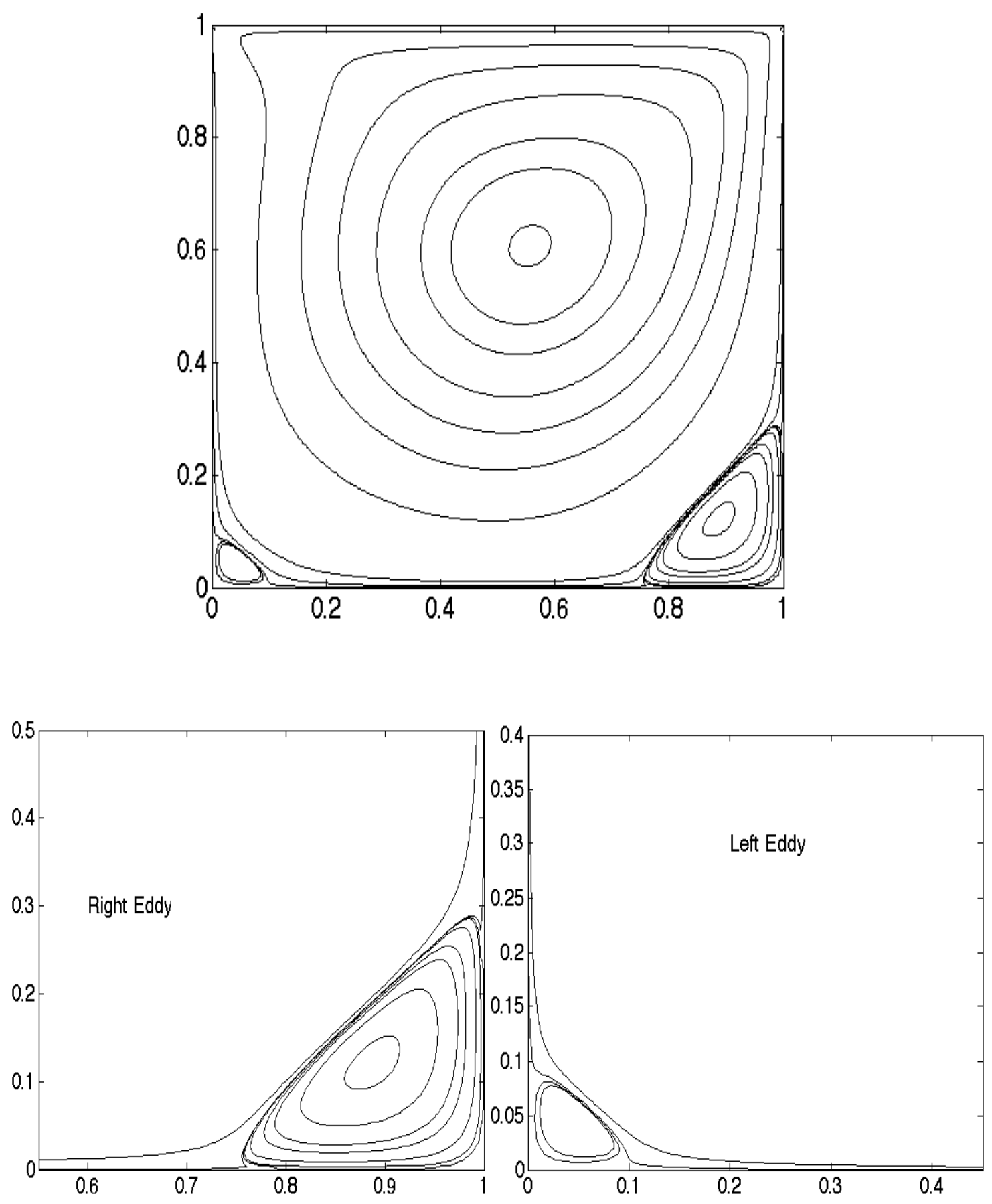

(a) 

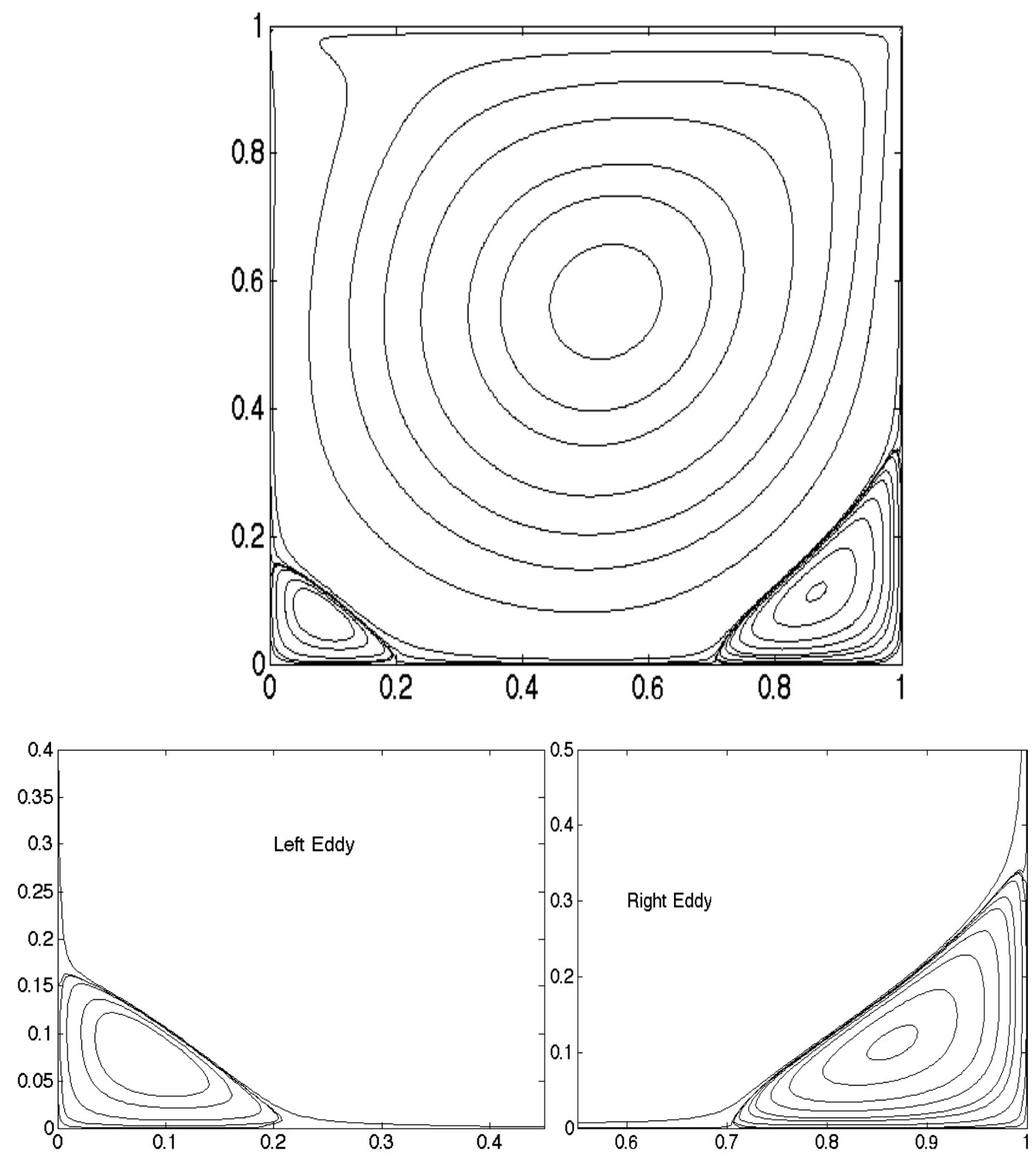

(b) 

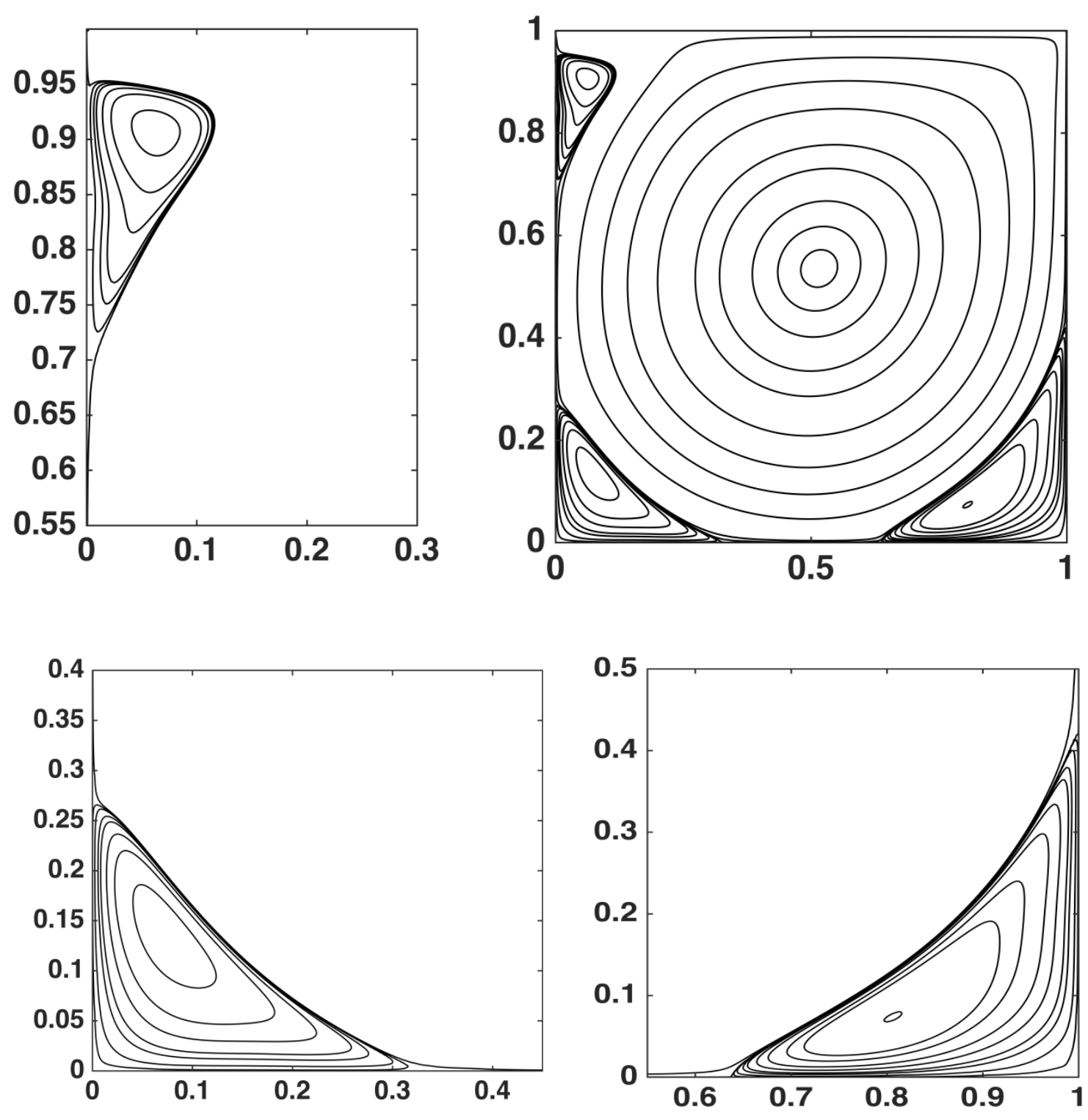

(c) 

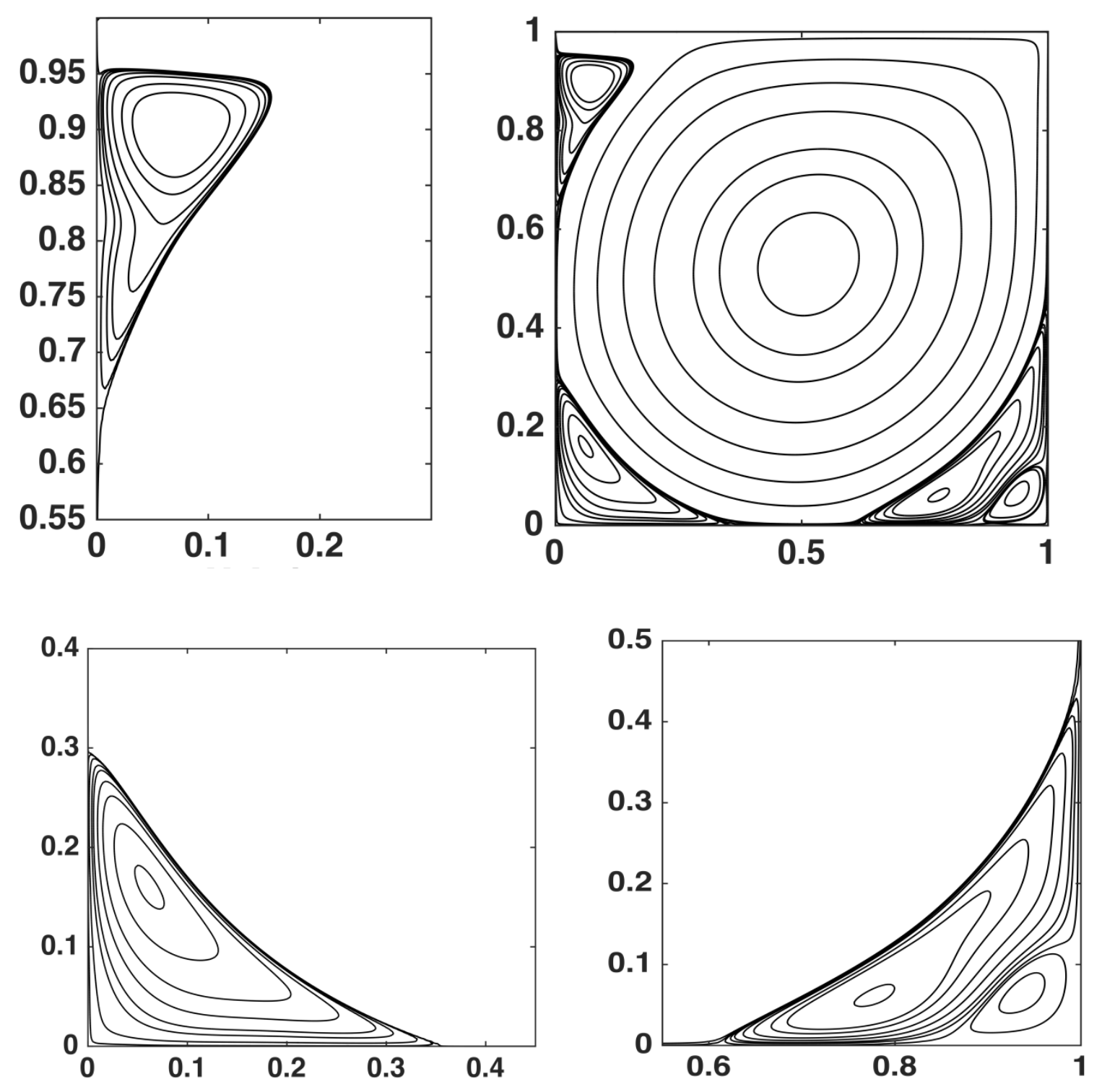

(d)

Fig. 4 

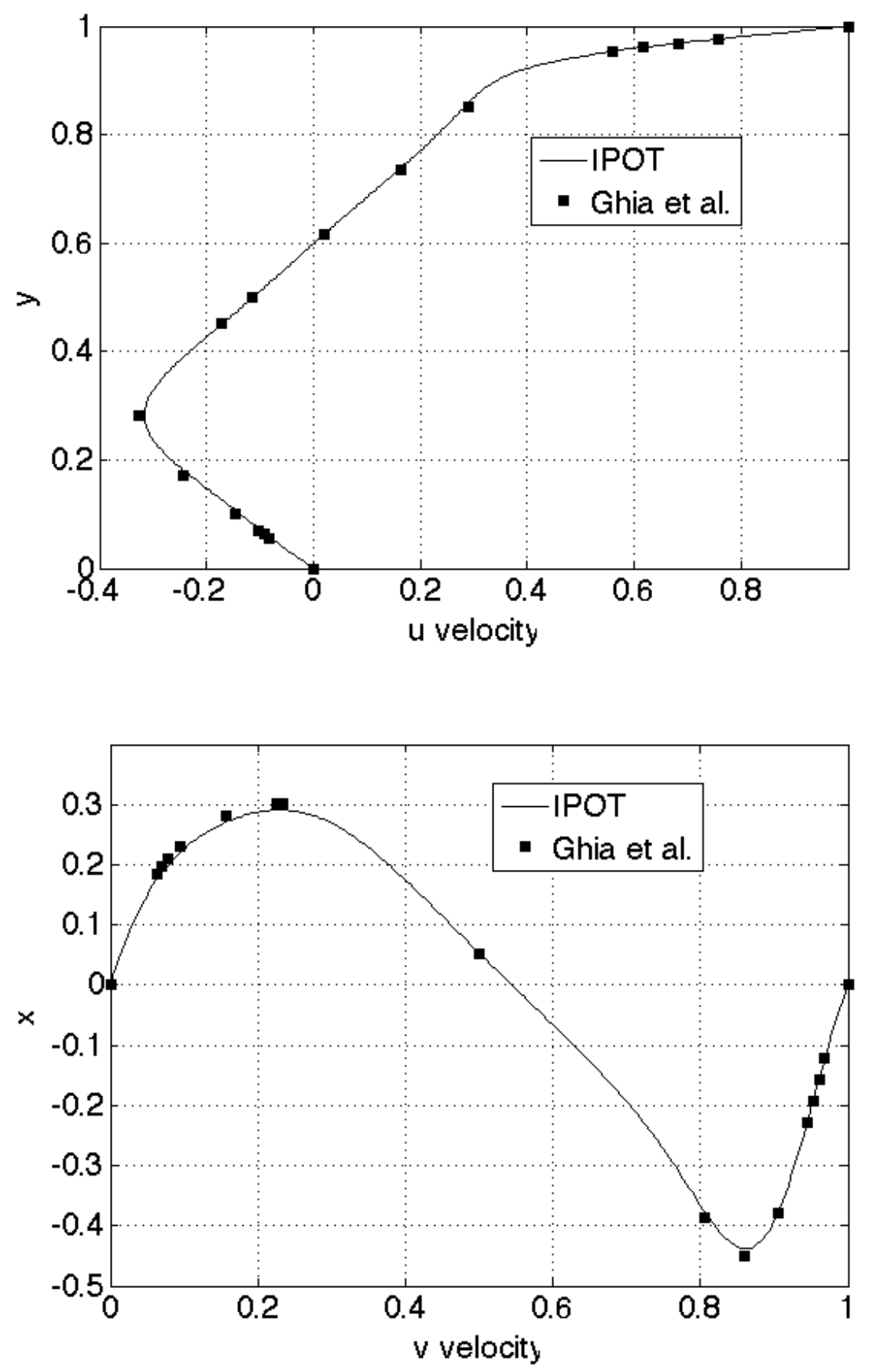

(a) 

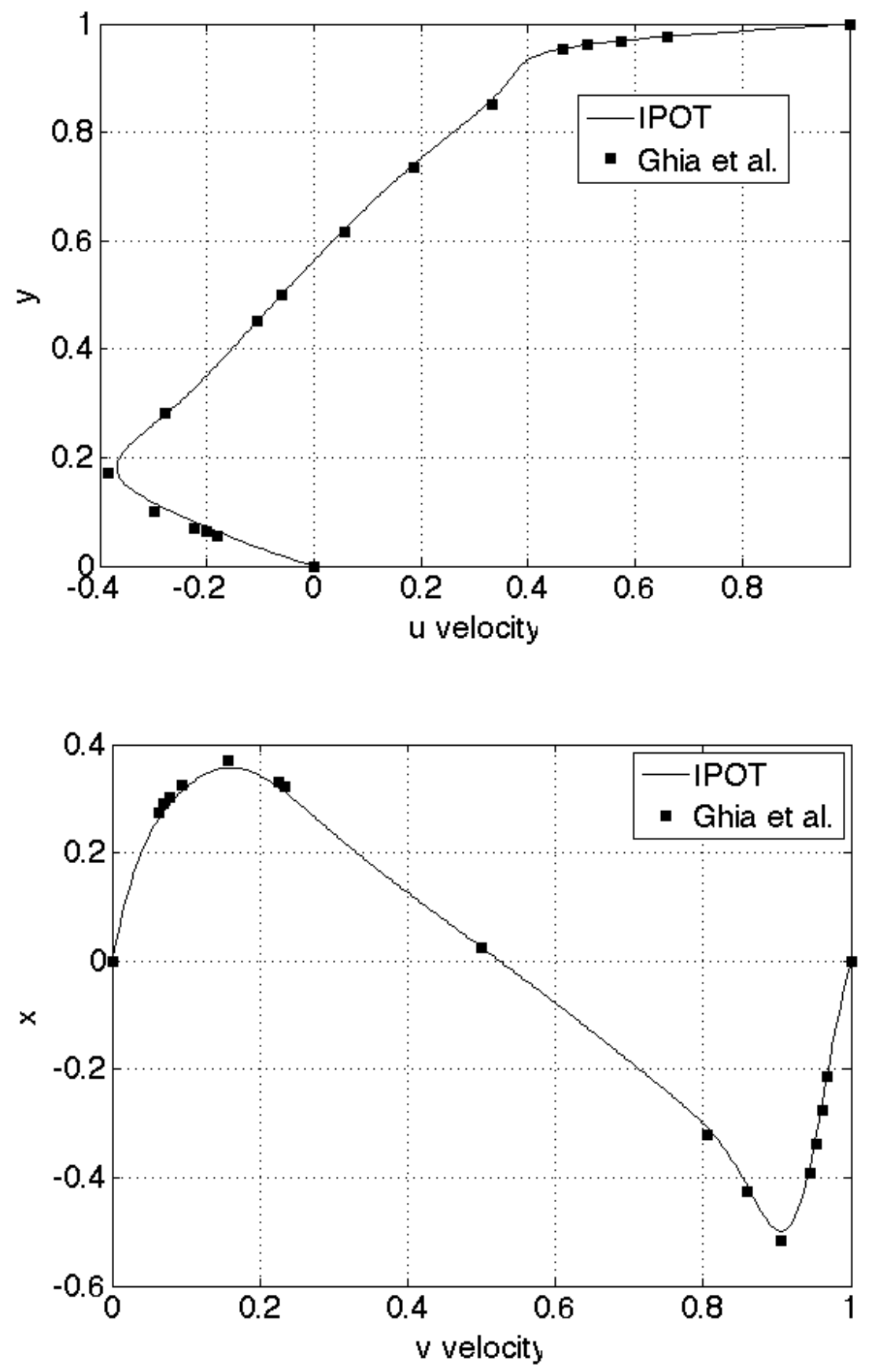

(b) 

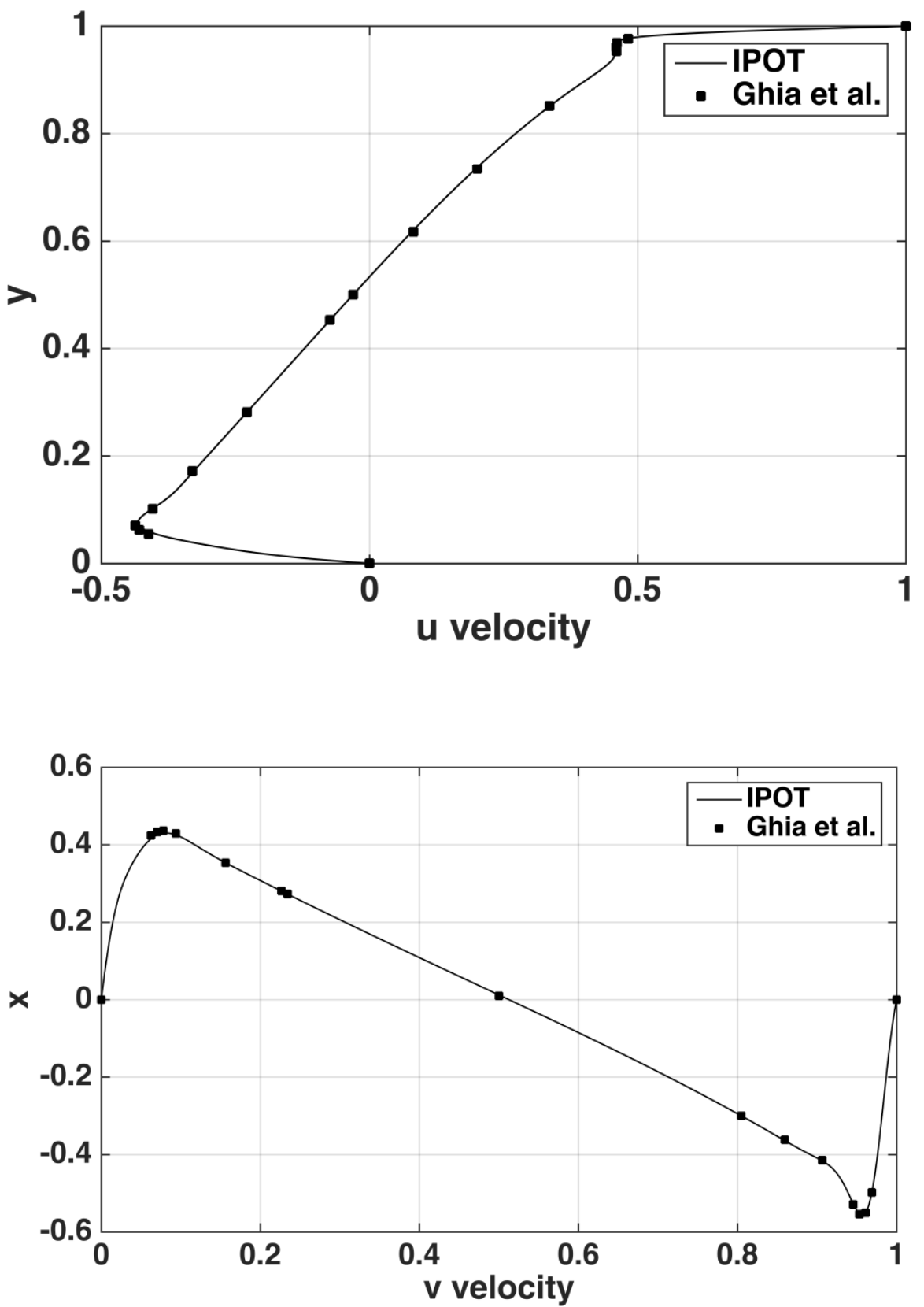

(c) 

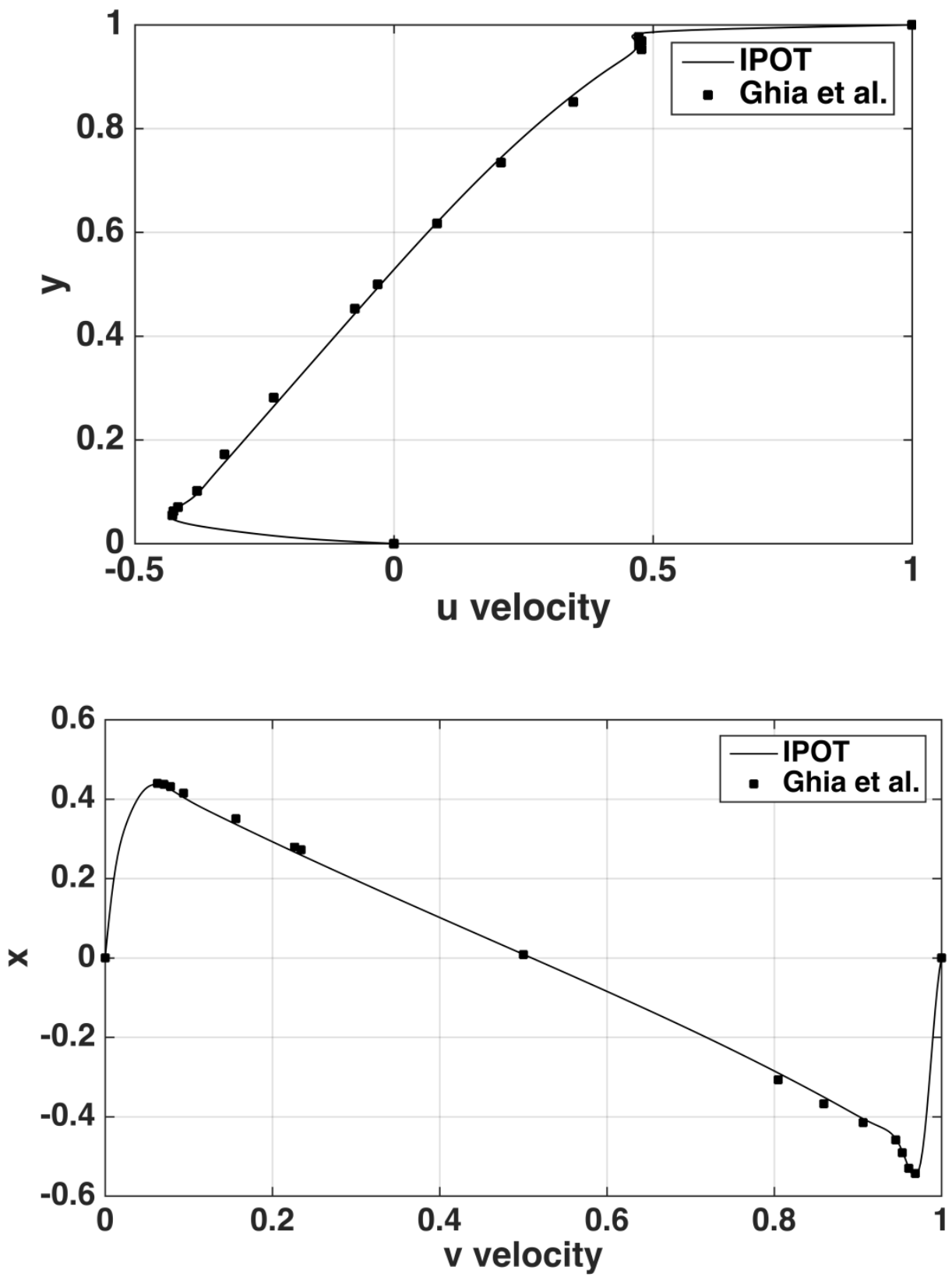

(d)

Fig. 5 


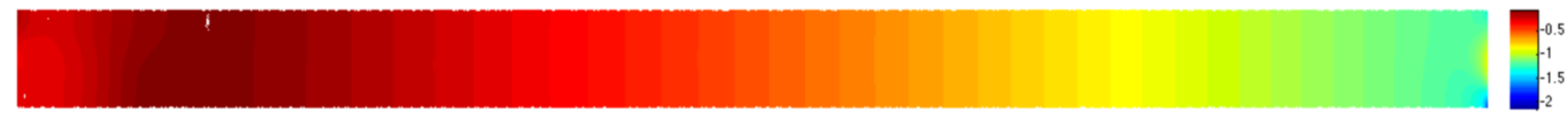

(a)

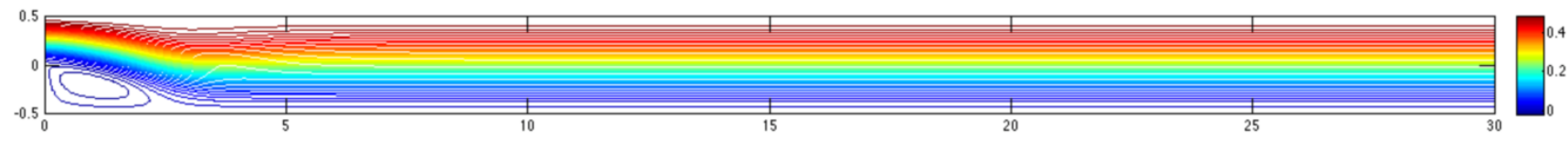

(b)

Fig. 6 

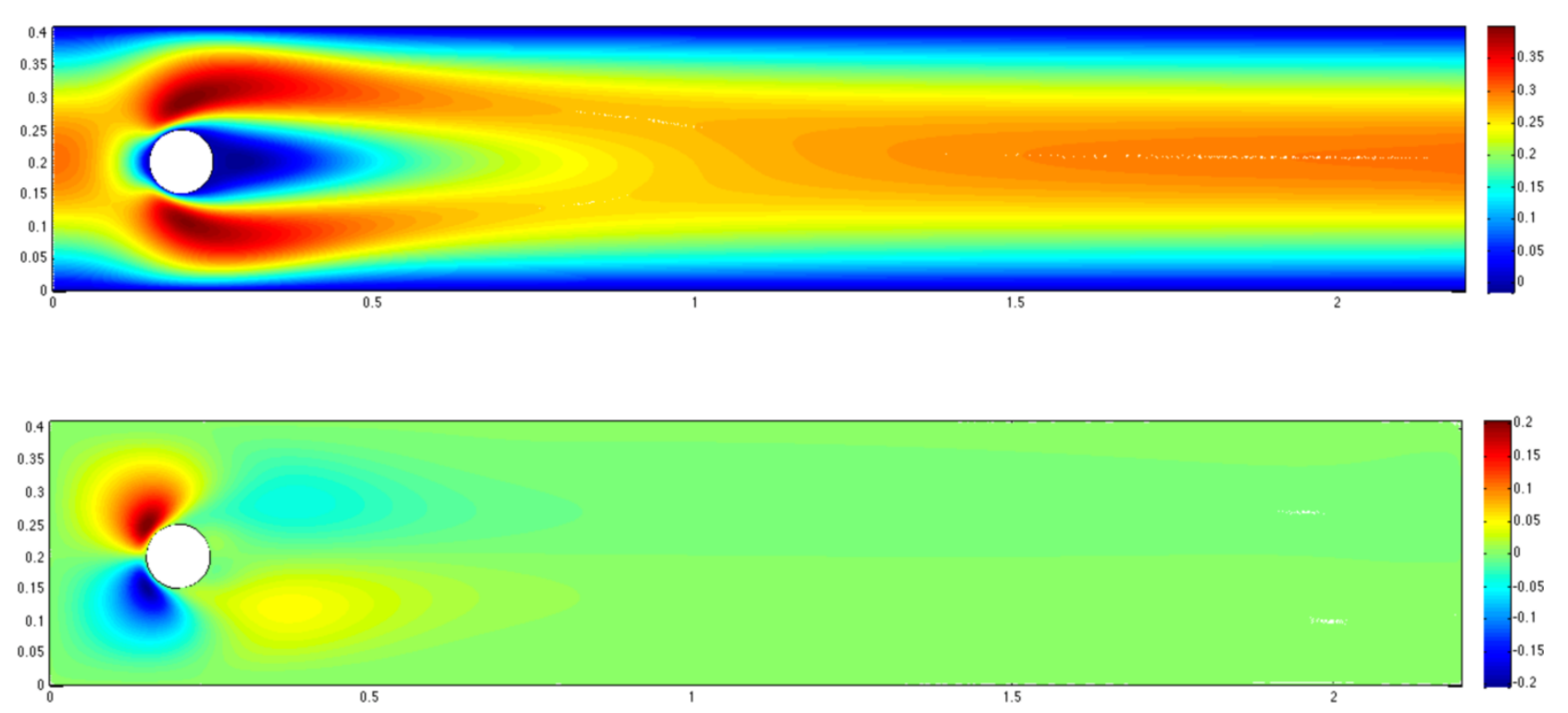

(a)
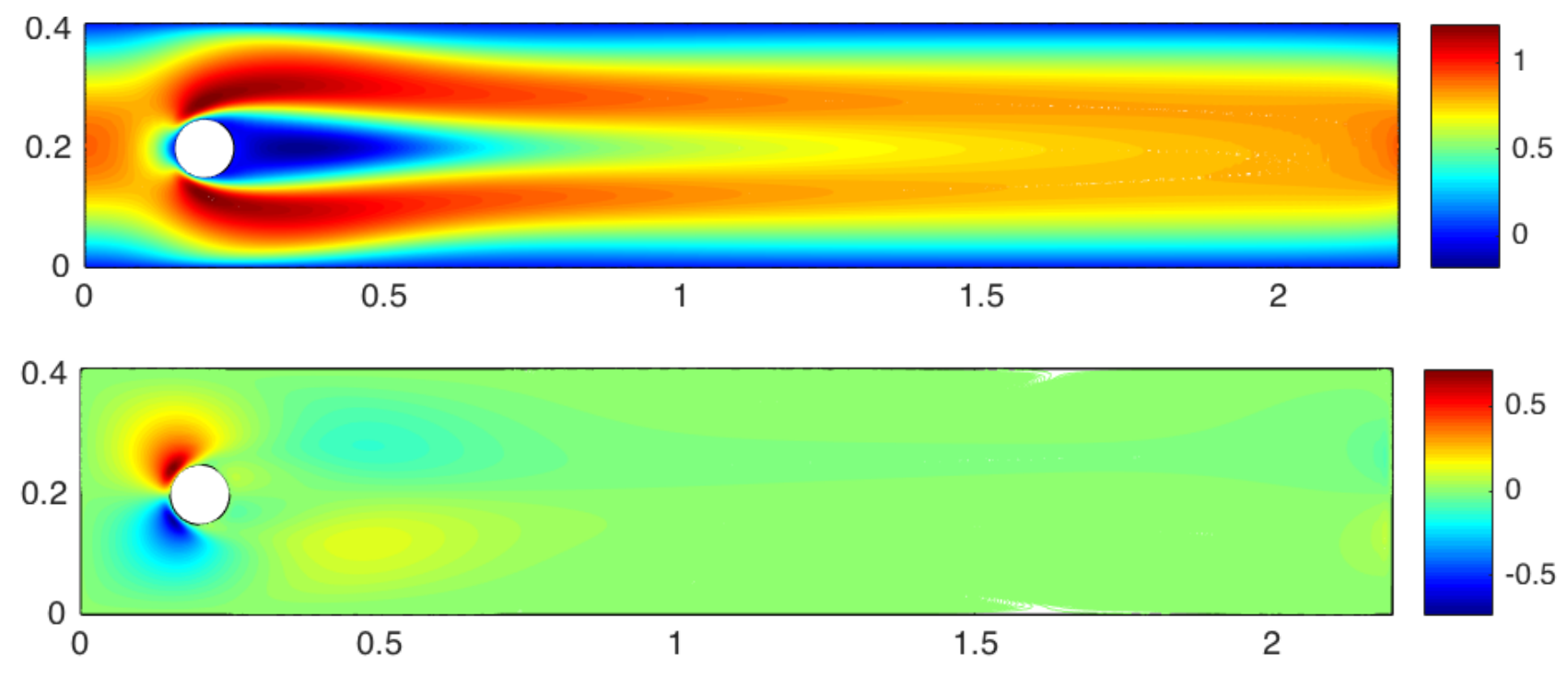

(b)

Fig. 7 


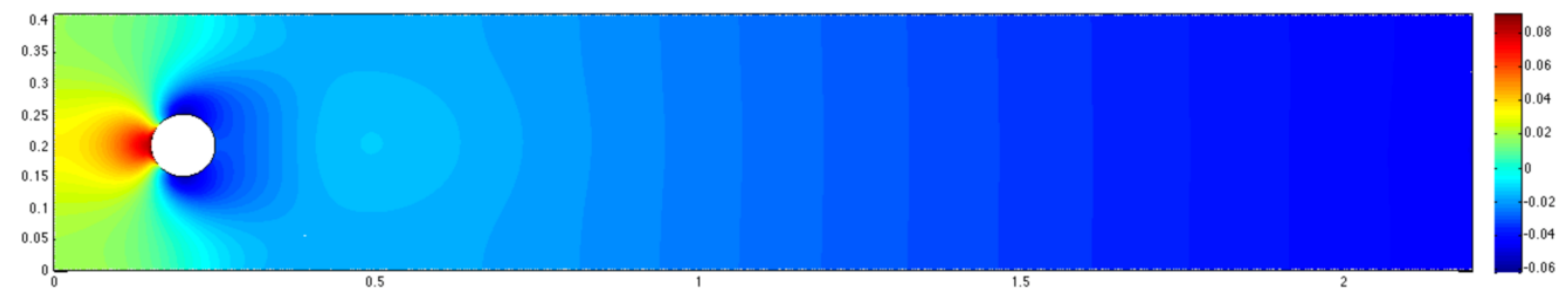

(a)

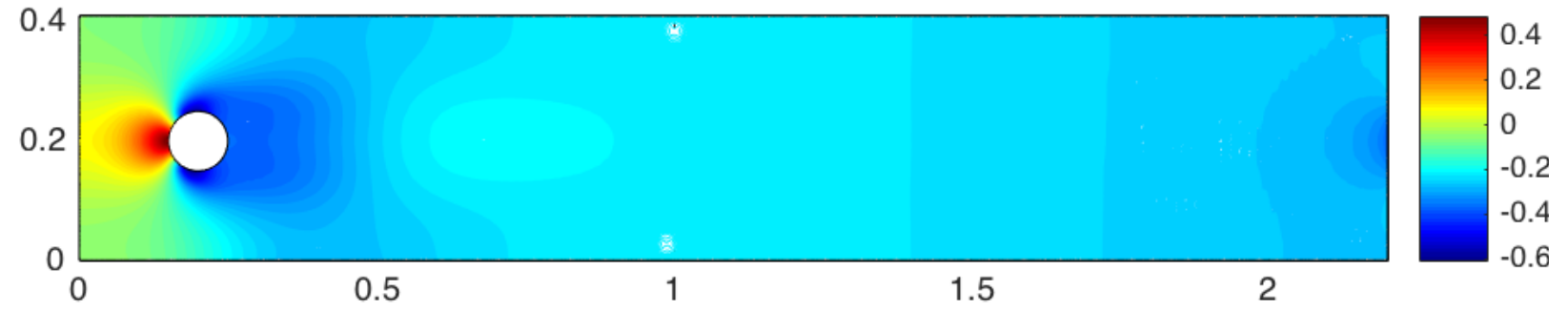

(b)

Fig. 8 


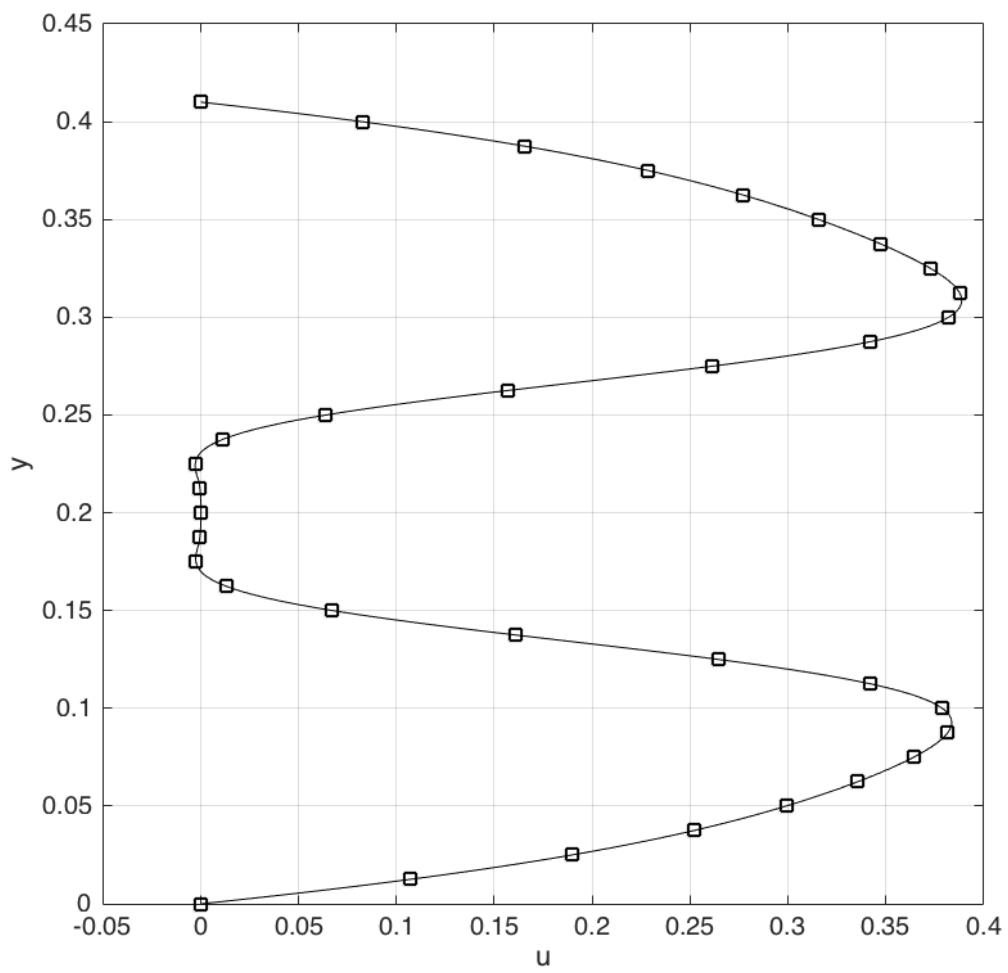

(a)

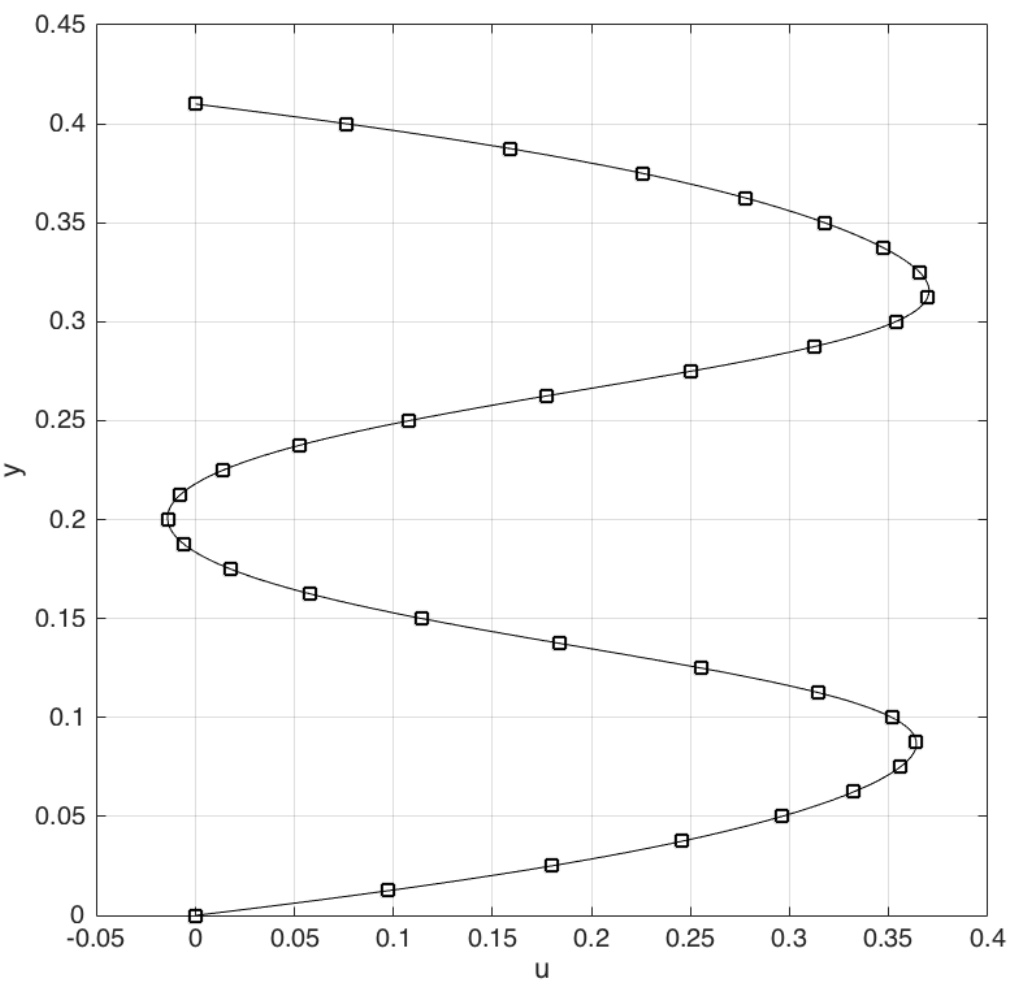

(b)

Fig. 9 

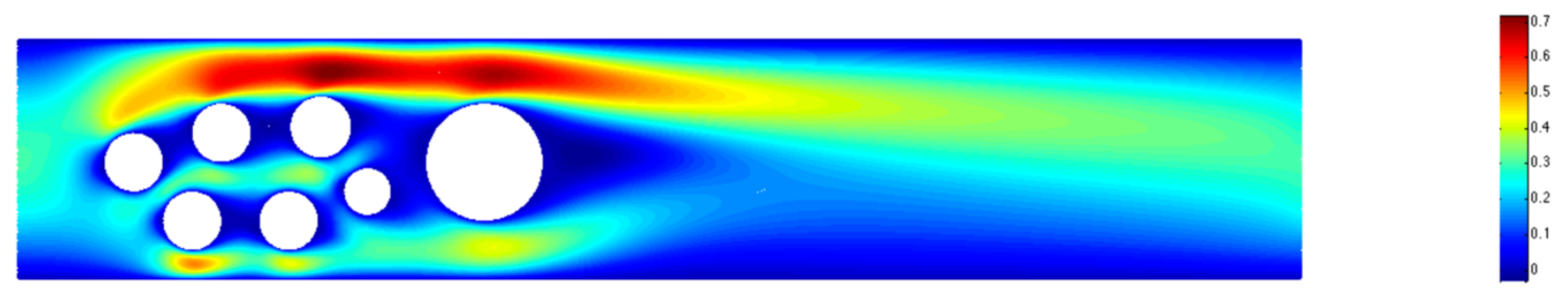

(a)
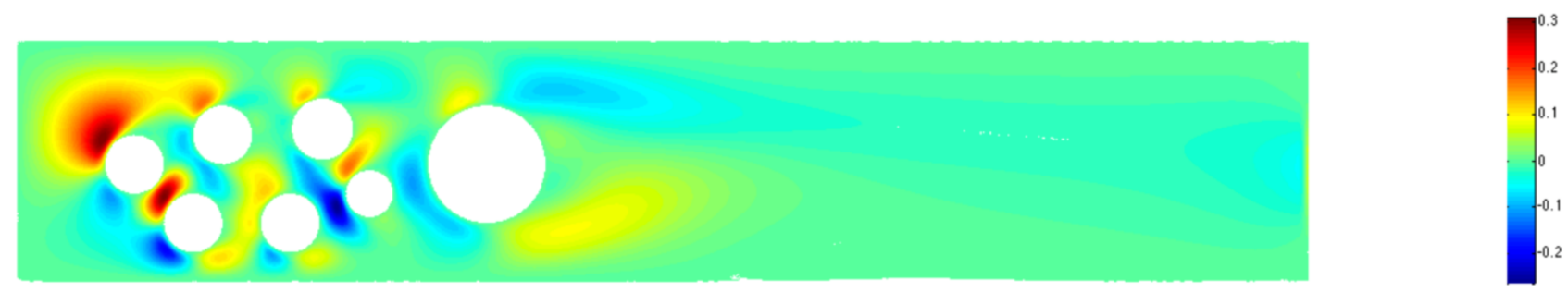

(b)

Fig. 10 


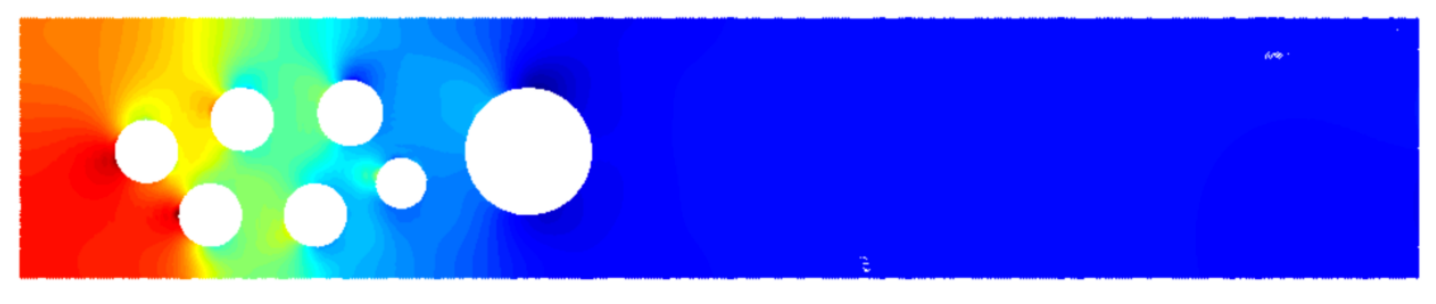

Fig. 11 


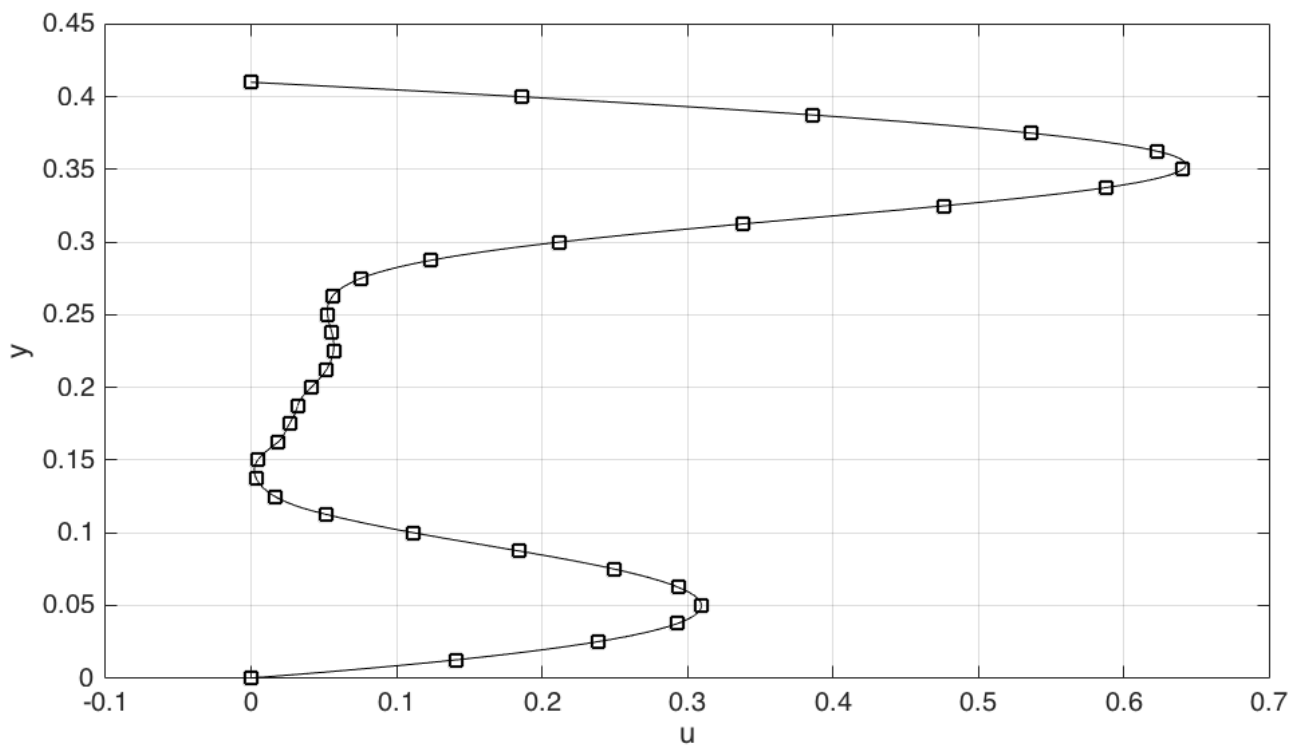

(a)

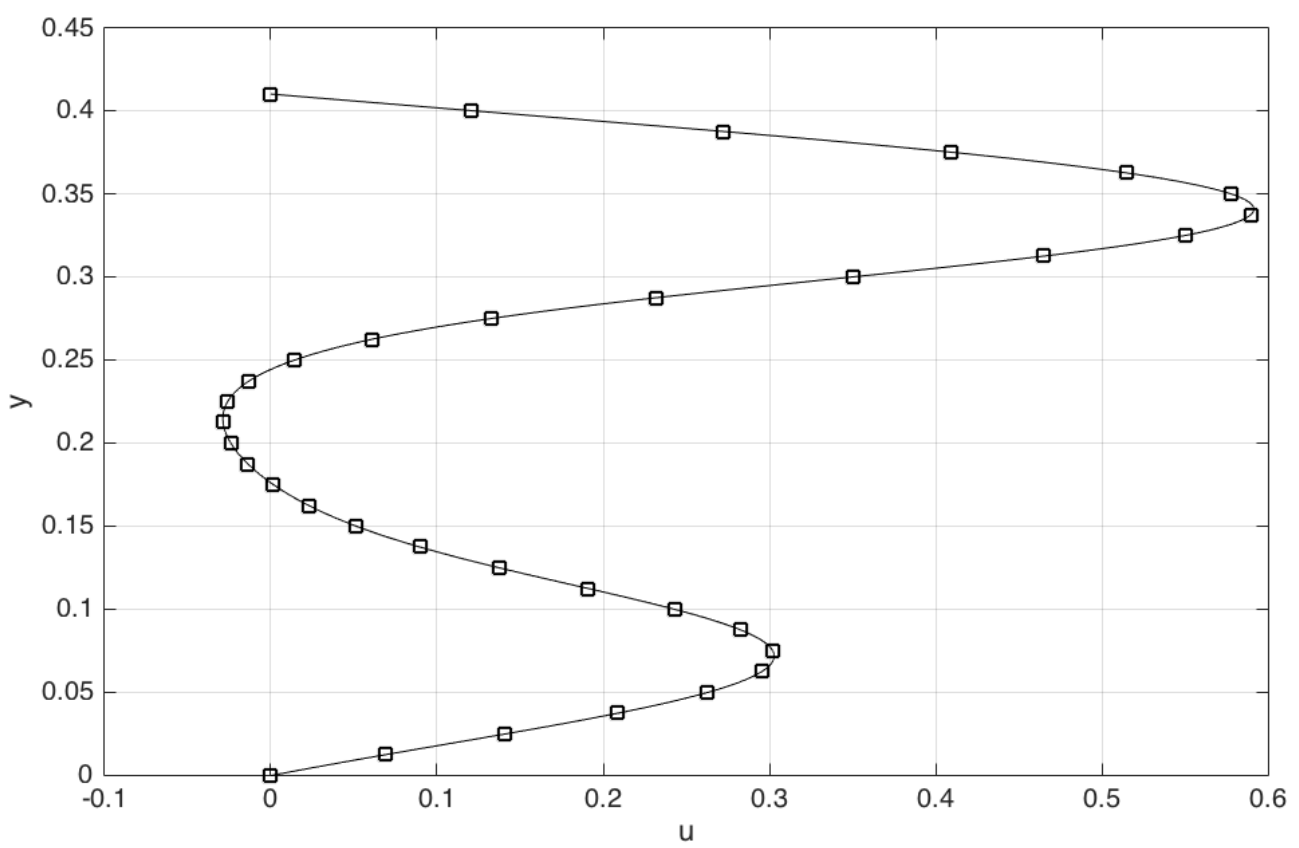

(b)

Fig. 12 


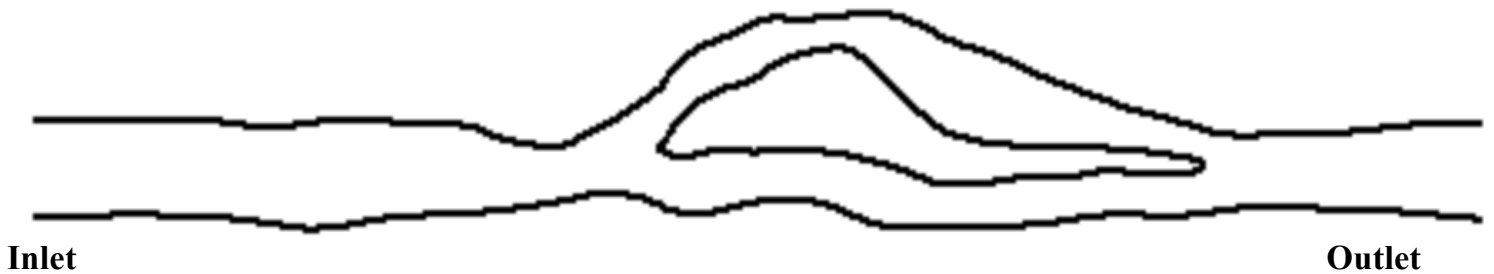

Fig. 13 


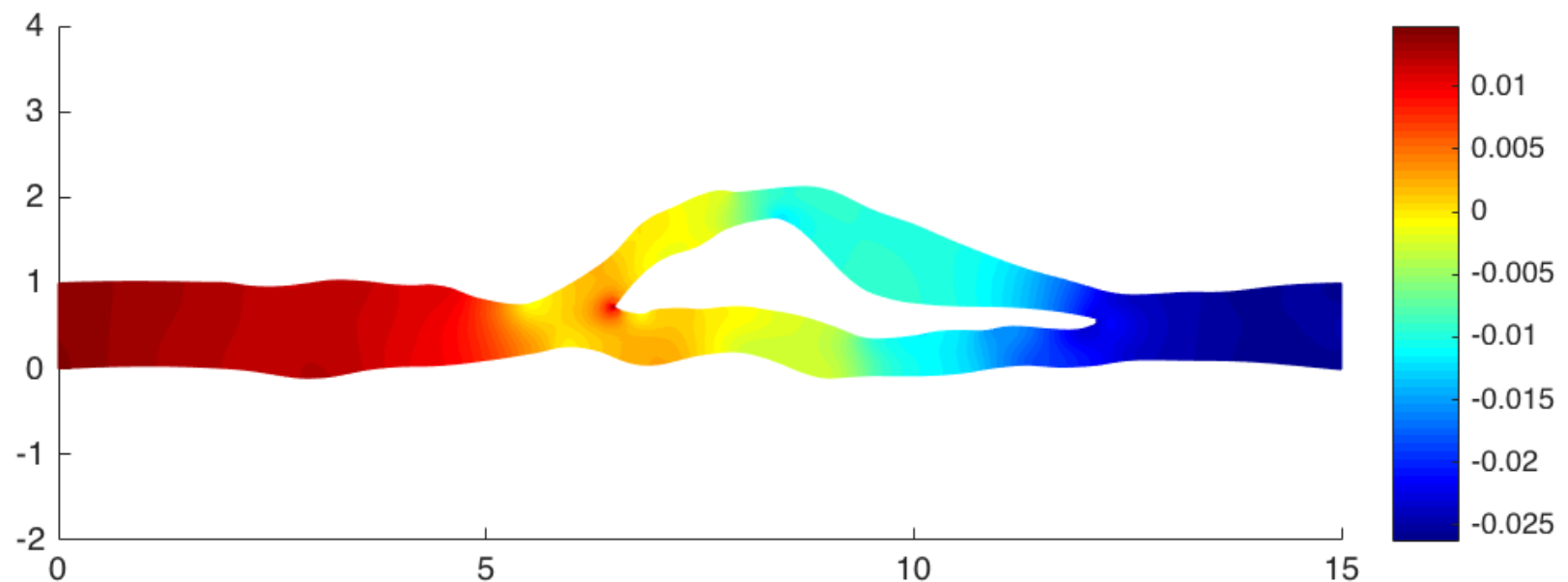

(a)

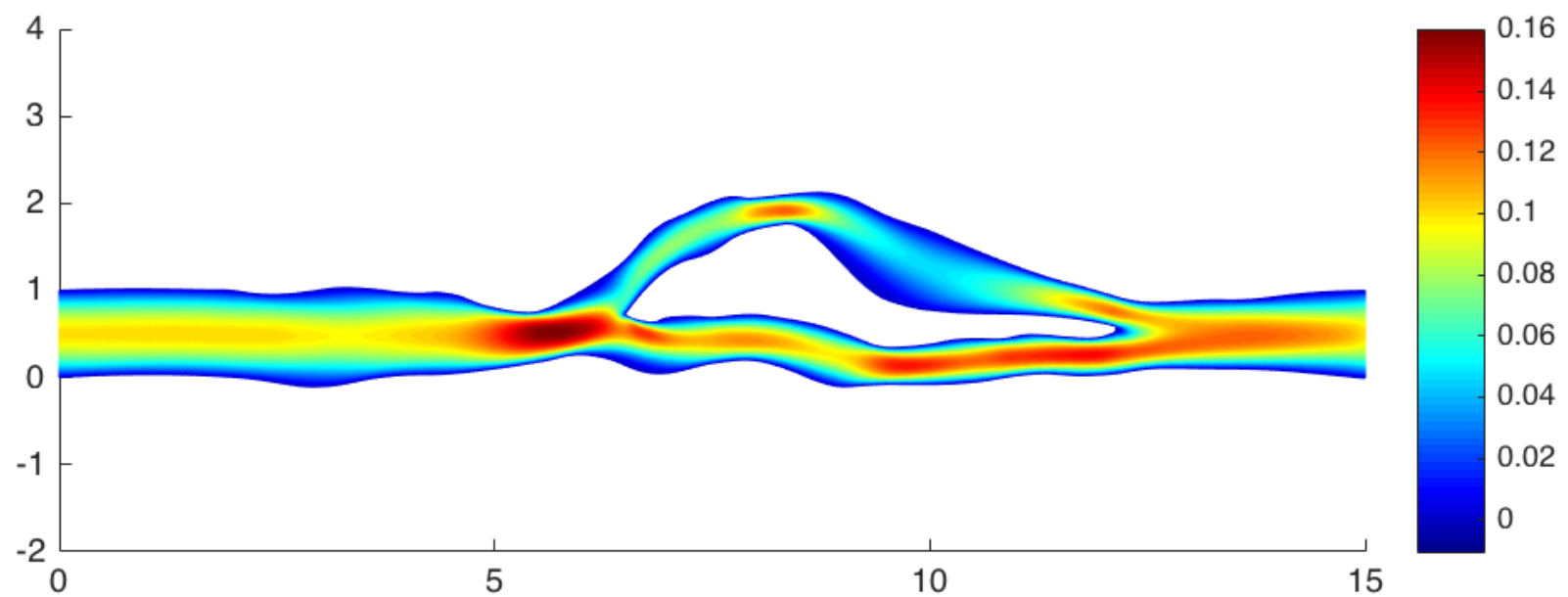

(b)

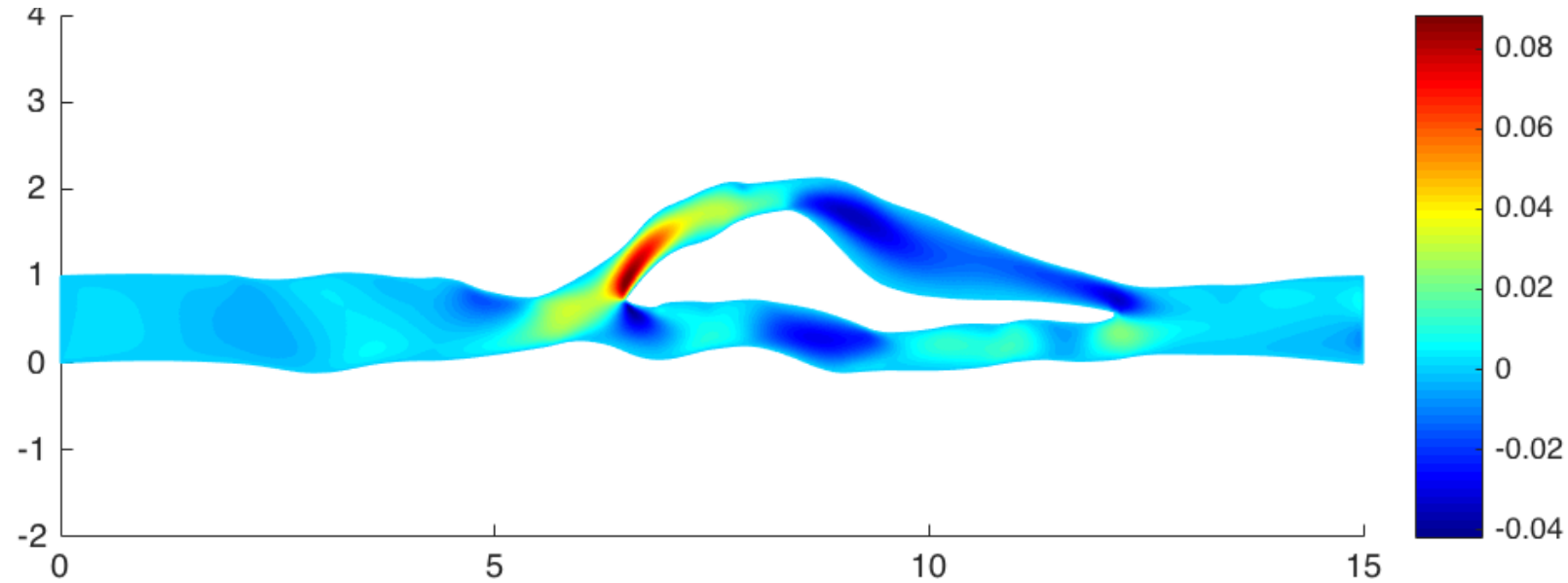

(c) 


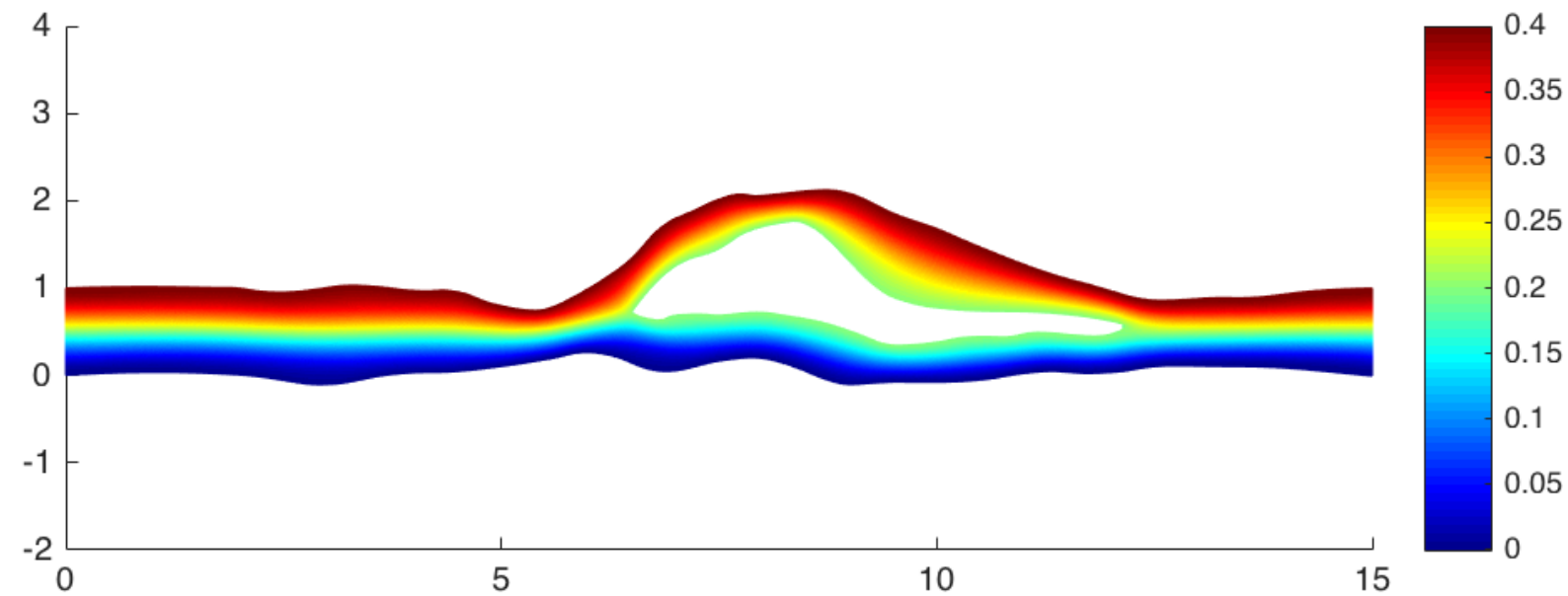

(d)

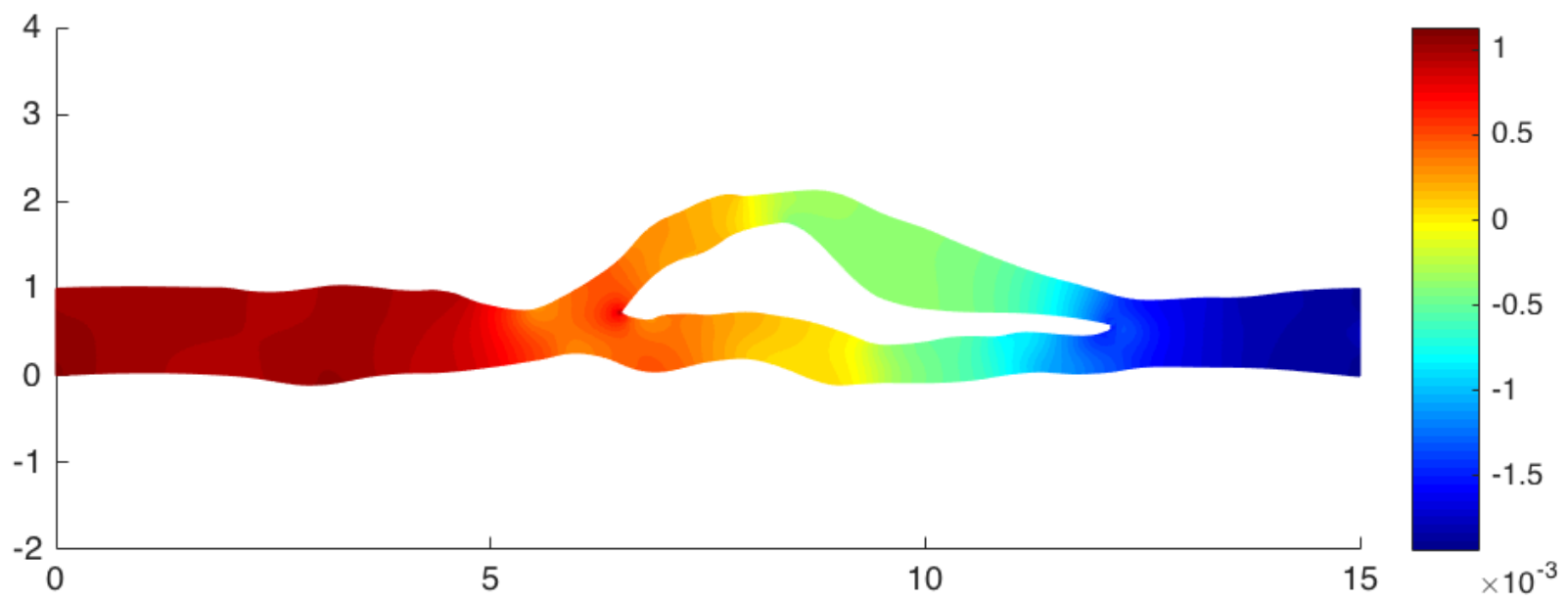

(e)

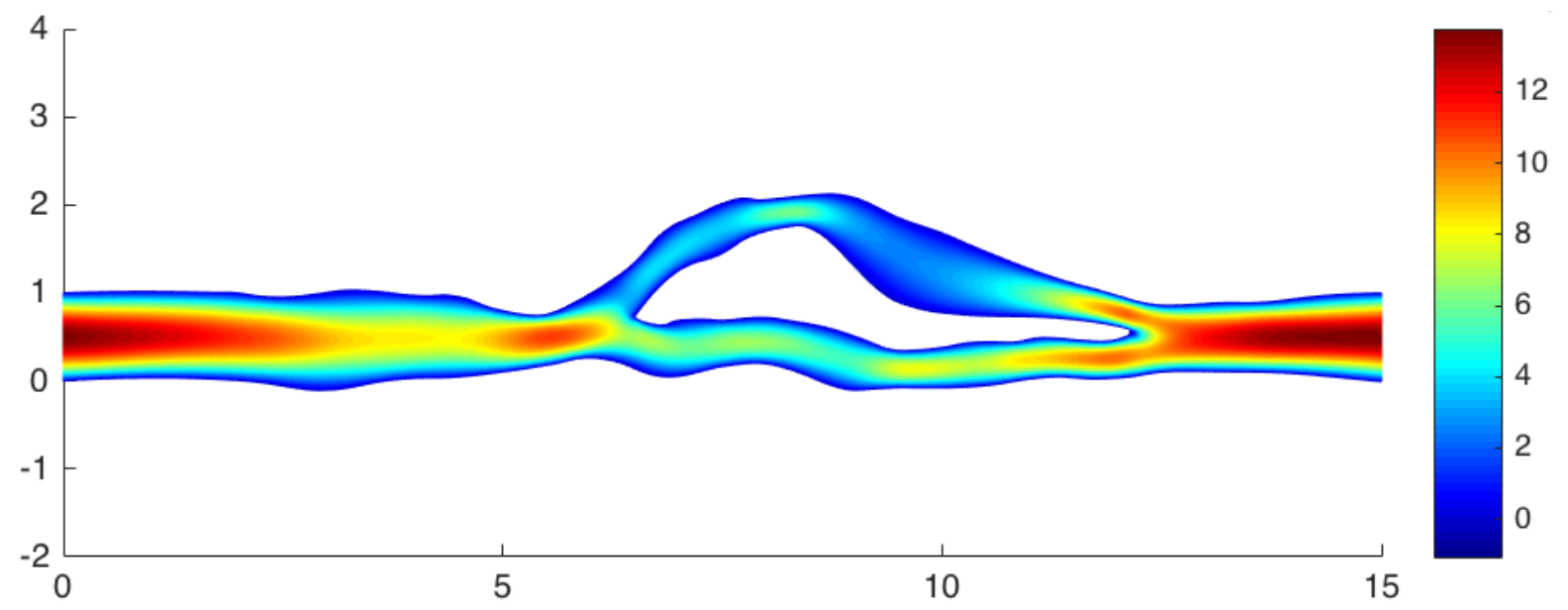

(f) 


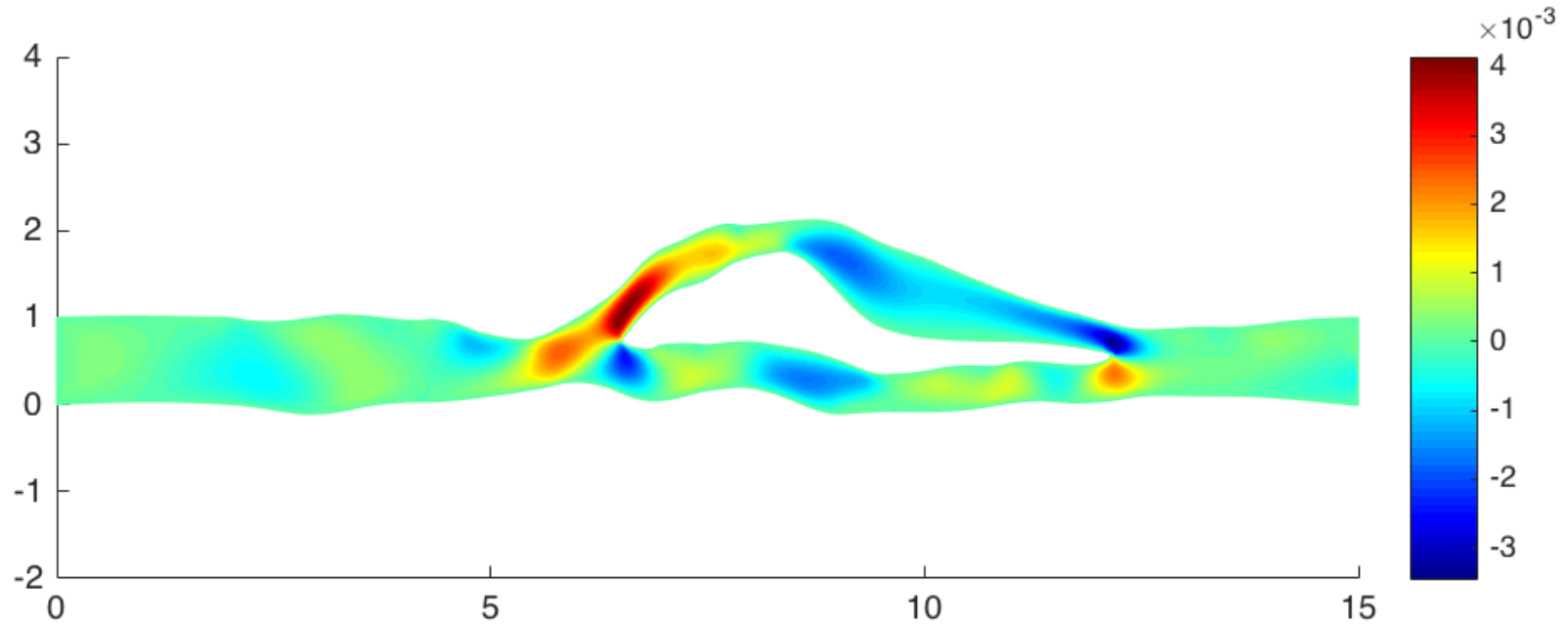

(g)

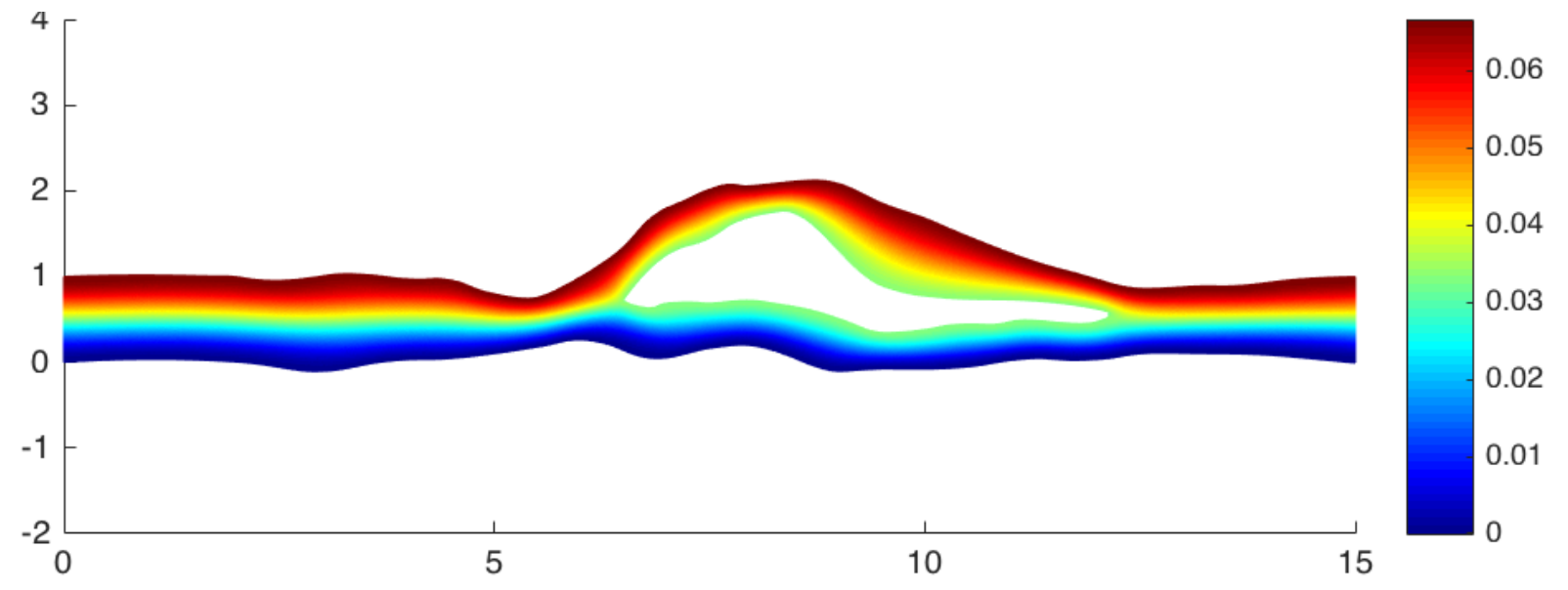

(h)

Fig. 14 


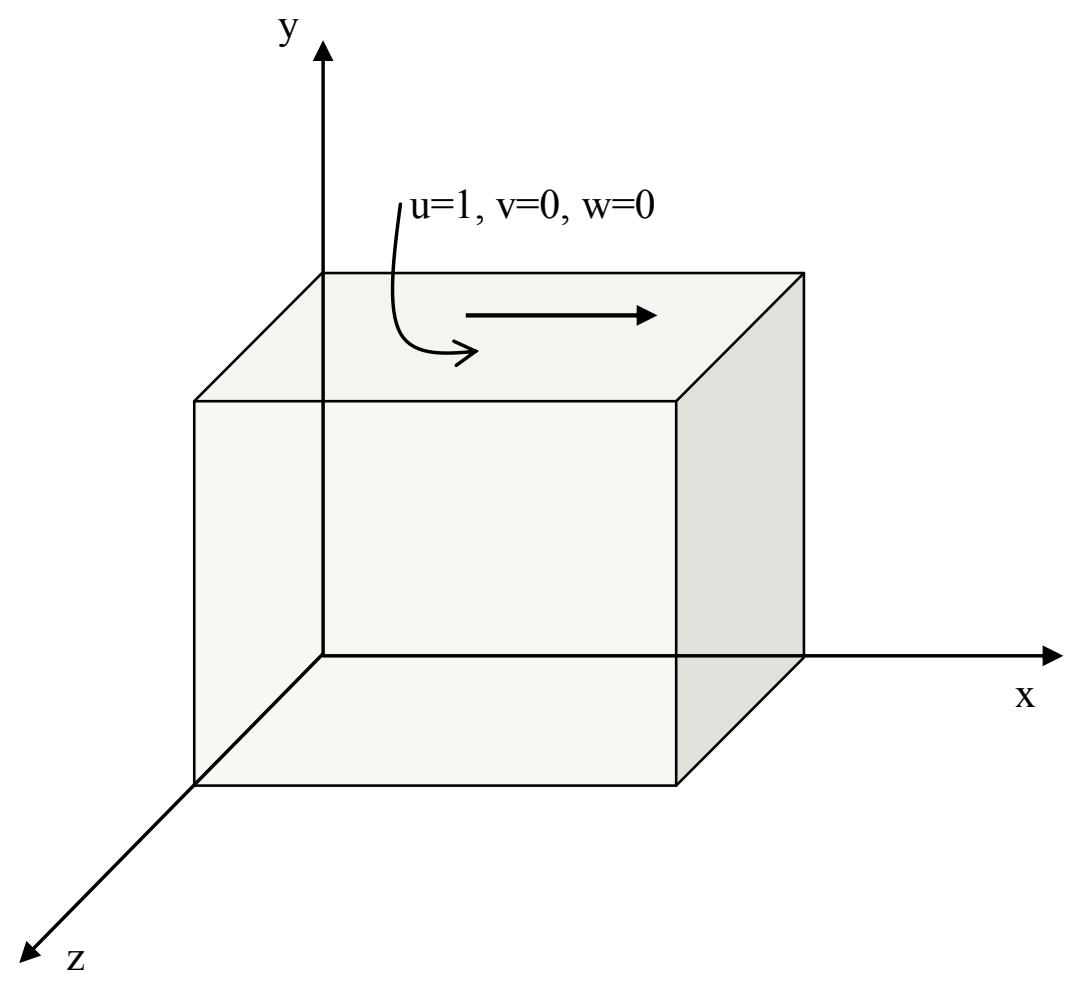

Fig. 15 


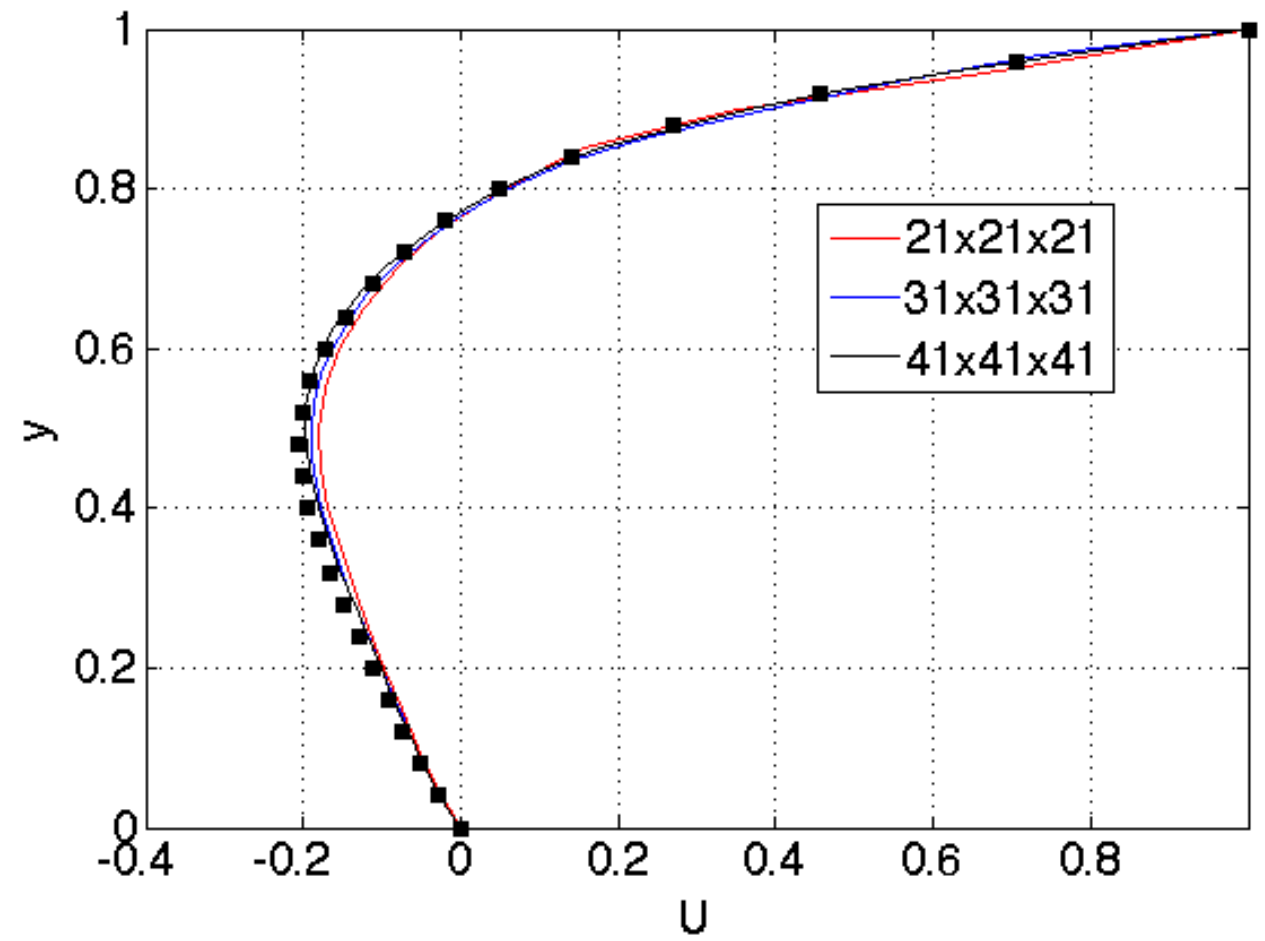

(a)

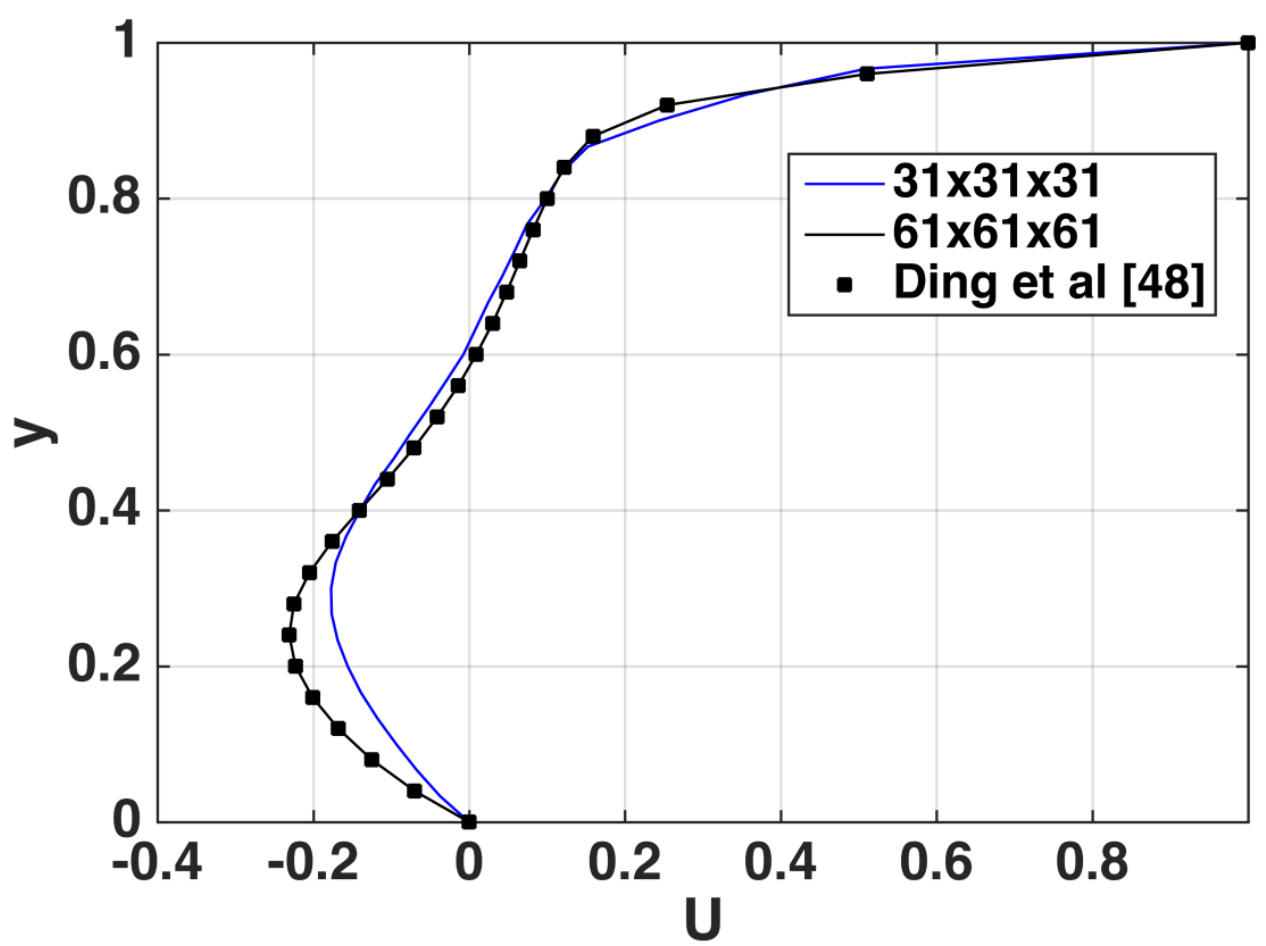

(b)

Fig. 16 


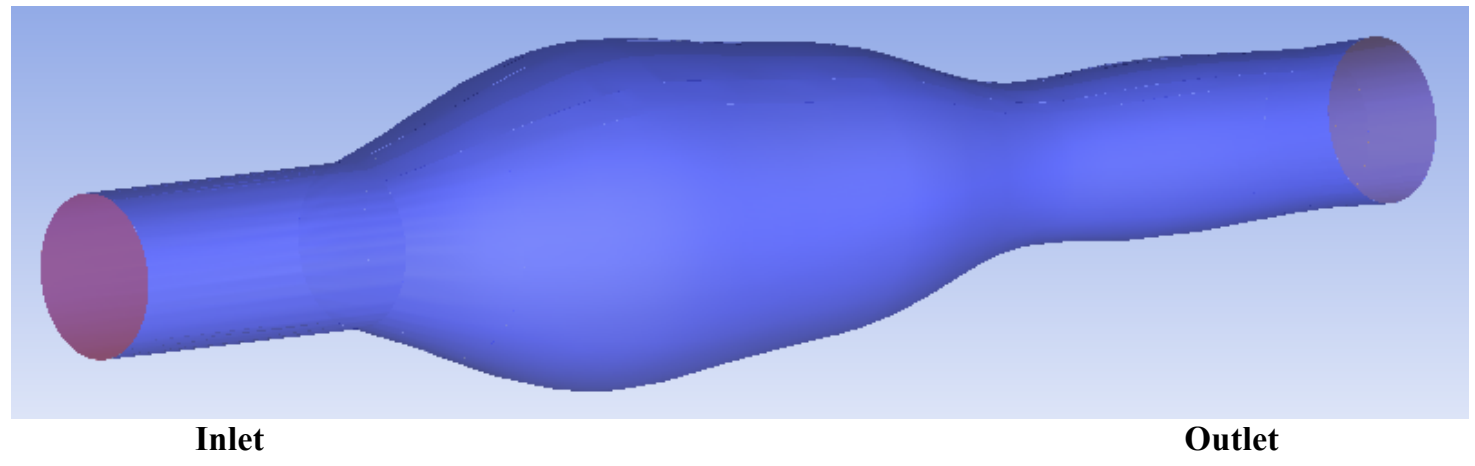

Fig. 17 


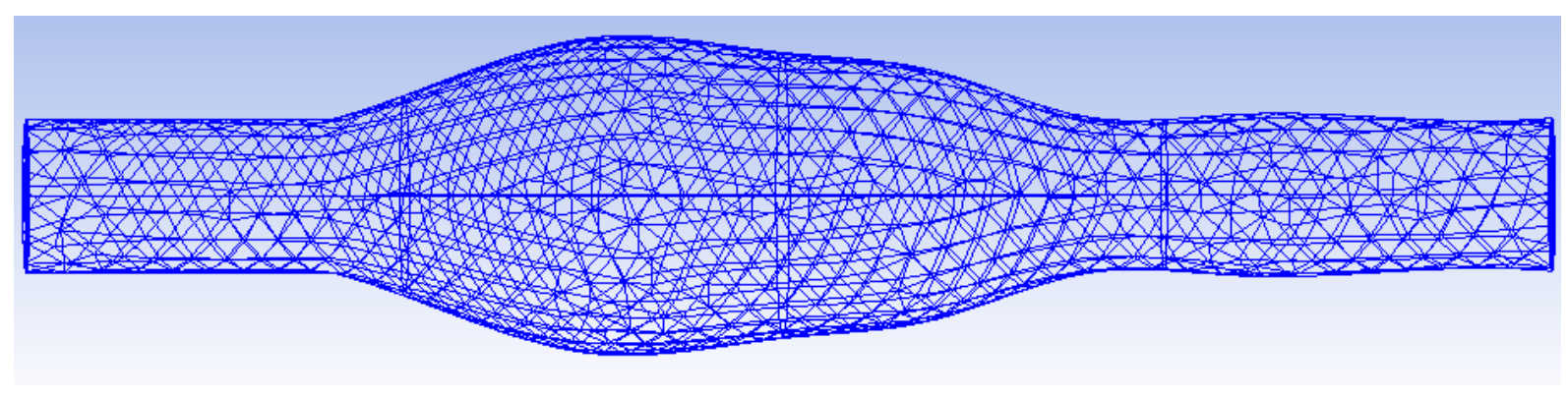

(a)

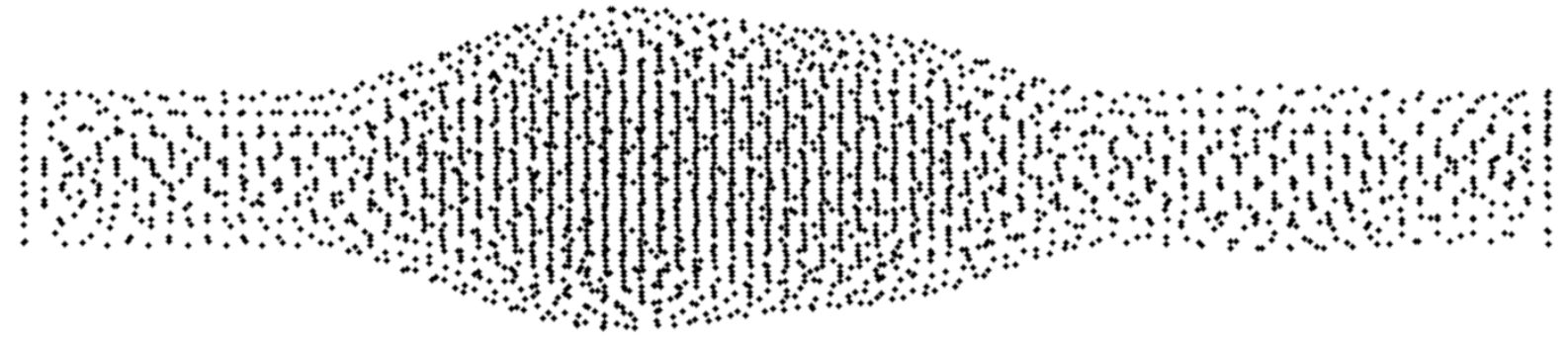

(b)

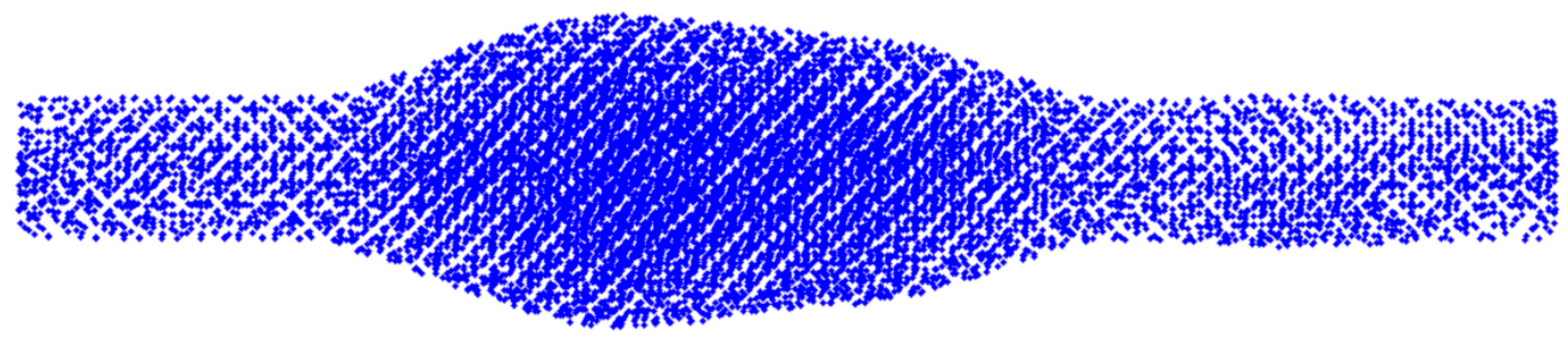

(c)

Fig. 18 


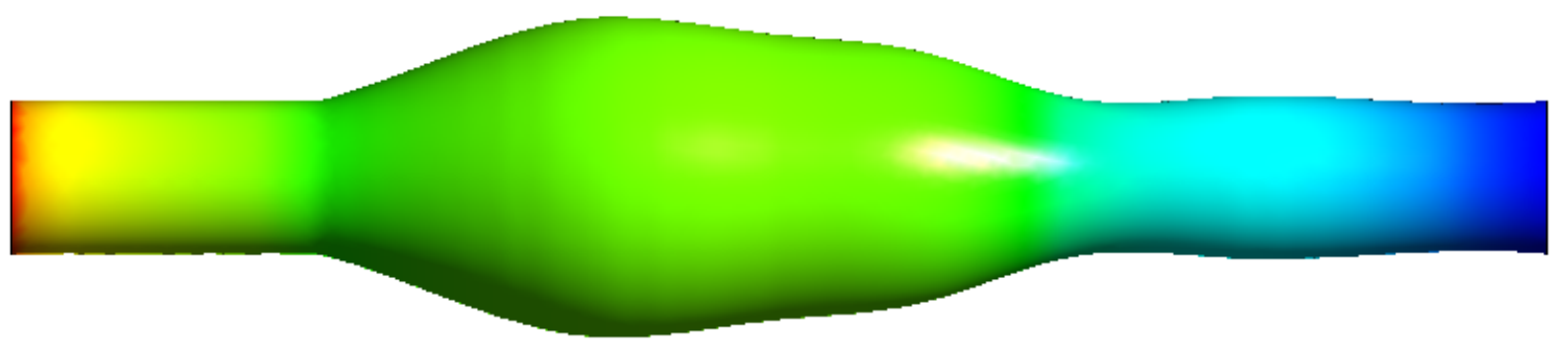

(a)

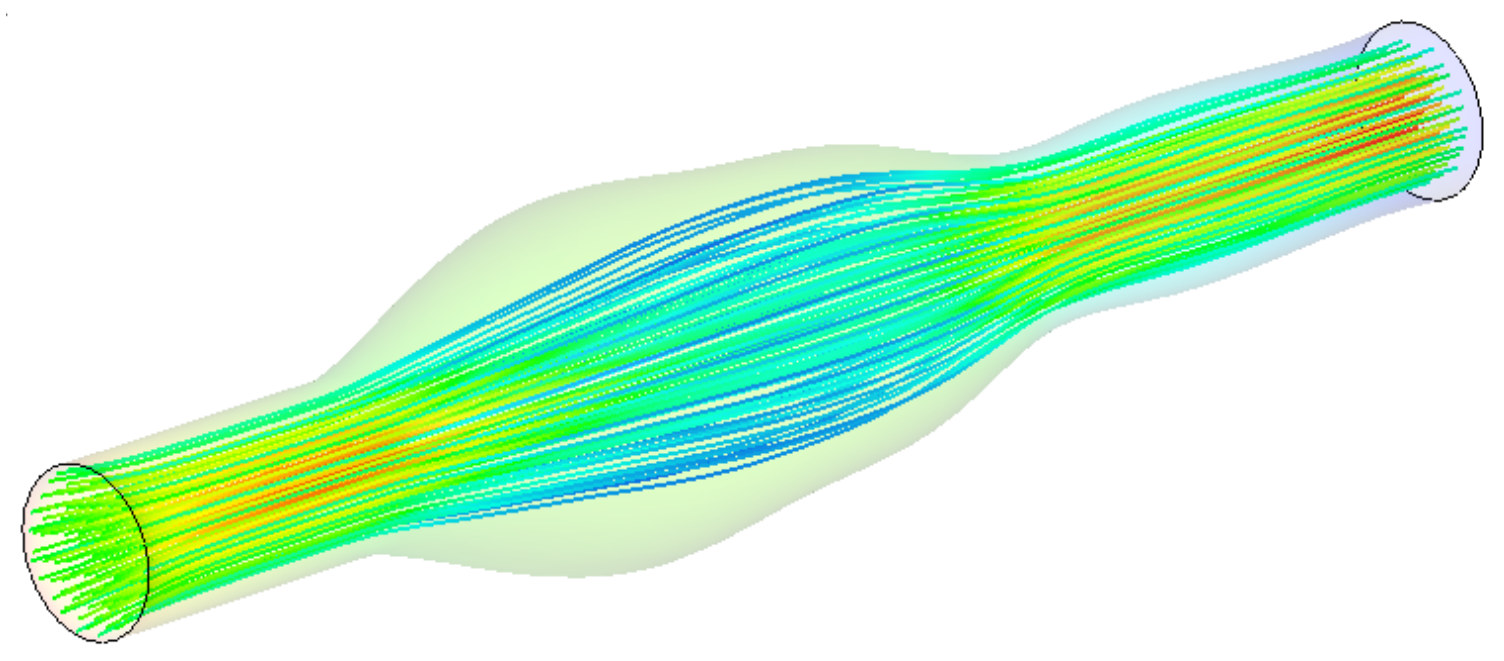

(b)

Fig. 19 